Pacific Journal of Mathematic 


\title{
SPECTRAL THEORY II. RESOLUTIONS OF THE IDENTITY
}

\author{
NELSON DUNFORD
}

\section{Introduction}

In attempting to extend elementary divisor theory to the case of a linear operator on a complex Banach space one is naturally led to a consideration of the various equivalent definitions of the multiplicity $\nu(\lambda)$ of a complex number $\lambda$ as a root of the minimal equation of a finite matrix $T$. Of the numerous equivalent definitions of this integer we have found only one which seems to have some virtue when applied to the infinite dimensional case. That one is as follows: $\nu(\lambda)$ is the smallest positive integer or zero for which

$$
|\xi-\lambda|^{\nu(\lambda)}\left|(\xi-T)^{-1}\right|
$$

is bounded for $\xi$ near $\lambda$. Thus the rate of growth of the resolvent

$$
T(\xi)=(\xi-T)^{-1}
$$

for $\xi$ near $\lambda$ determines $\nu(\lambda)$. In this paper we consider the problem of determining conditions on the rate of growth and the mean rate of growth of the resolvent which are necessary and sufficient for a complete reduction of a linear operator on a complex Banach space. What is to be meant by a "complete" reduction? There are several apparent meanings that might be given to the notion of the resolution of the identity for an operator, all reducing to the classical one in the case of a finite matrix. For example, are we to require that $E_{\sigma}$ be defined for all Borel sets $\sigma$ or for $\sigma$ in some sufficiently large subalgebra; should it be countably or just finitely additive; should it be bounded or not? All problems are legitimate and in this paper we have chosen the most restrictive of all the obvious interpretations. Consequently the conditions found on $T(\xi)$ are restrictive and the corresponding class of operators is small. On the other hand, such operators have many important properties not shared by operators outside this class. Other meanings for the notion of resolution of the identity will be

Received January 2, 1951. The research contained in this paper was done under contract N7onr-448 with, and reported to, the Office of Naval Research during the period between September 1, 1947, and May 1, 1948.

Pacific J. Math. 2 (1952), 559-614 
considered in another report.

Before stating what is to be meant by a resolution of the identity for $T$, let us recall that if $T$ is a linear operator in the finite dimensional linear vector space $X$ over the field of complex numbers, and $\Pi_{i}\left(\lambda-\lambda_{i}\right)^{\nu_{i}}\left(\lambda_{i}\right.$ distinct $)$ is its minimal polynomial, then there are projections $E_{\lambda_{i}}$ with

$$
E_{\lambda_{i}} X=\left[x \mid\left(T-\lambda_{i}\right)^{\nu_{i}} x=0\right]
$$

and such that

$$
l=E_{\lambda_{1}}+\cdots+E_{\lambda_{k}}
$$

If, for a Borel set $\sigma$ in the complex plane, $E_{\sigma}$ is defined to be the sum of those $E_{\lambda_{i}}$ for which $\lambda_{i} \in \sigma$, then $E_{\sigma}$ is a resolution of the identity for $T$ in the sense that it has the properties (i) below:

$$
\left\{\begin{array}{l}
E_{\sigma} E_{\delta}=E_{\sigma \delta}, E_{\sigma^{\prime}}=I-E_{\sigma}, T E_{\sigma}=E_{\sigma} T \\
E_{\sigma} x \text { is completely additive in } \sigma, x \in X \\
\text { the spectrum of } T \text { when considered as an operator in } E_{\sigma} X \text { is contained } \\
\text { in } \bar{\sigma}, \text { the closure of } \sigma .
\end{array}\right.
$$

If, for a given linear operator $T$ in a complex Banach space, there exists a family $E_{\sigma}$ ( $\sigma$ a Borel set) of operators in $X$ satisfying ( $\left.i\right)$, then $E_{\sigma}$ is called a resolution of the identity for $T$. Such operators will be called spectral operators. If $T$ is a spectral operator its resolution of the identity is unique, and operators $f(T)$ corresponding to scalar functions analytic and single valued on the spectrum $\sigma(T)$ are given by the formula

$$
f(T)=\sum_{n=0}^{\infty} \int_{\sigma(T)} \frac{f^{(n)}(\lambda)}{n !}(T-\lambda)^{n} d E_{\lambda},
$$

where the integral exists as a Riemann integral in the uniform topology of operators and the series is convergent in the uniform topology of operators.

The main problem is, however, to determine when $T$ is a spectral operator. We have endeavored to state conditions on the rate of growth and the mean rate of growth of the resolvent

$$
T(\xi)=(\xi-T)^{-1}
$$


which are sufficient and in some cases necessary and sufficient for the existence of a resolution of the identity. In order to do this, we have had to restrict ourselves to the case where the spectrum $\sigma(T)$ lies in a sufficiently smooth Jordan curve. To describe briefly in this introduction the nature of the results obtained in this direction, suppose that $T$ has its spectrum in the interval $[0,1]$. The underlying assumption is then that for each $\lambda \in[0,1]$ there is a positive integer $\nu(\lambda)$ and a positive number $M(\lambda)$ such that

$$
\left|\mu^{\nu(\lambda)} T(\lambda+i \mu)\right| \leq M(\lambda), \quad 0<|\mu|<1
$$

This alone is far from sufficient to ensure that $T$ is a spectral operator, even in case

$$
\nu(\lambda)=M(\lambda)=1
$$

An obvious necessary condition may be stated in terms of the following notion of residue. Let $C$ be a rectifiable Jordan curve contained in the set where $x^{*} T(\xi) x$ is analytic. Let $\sigma$ be the set of all singularities of $x^{*} T(\xi) x$ which are inside $C$. Then

$$
\left(x^{*}, x\right)_{\sigma}=\frac{1}{2 \pi i} \quad \int_{C}^{-} x^{*} T(\xi) x d \xi
$$

is called a residue of $x^{*} T(\xi) x$. It is clear that if $T$ has a resolution of the identity then

$$
\left(x^{*}, x\right)_{\sigma}=x^{*} E_{\sigma} x
$$

and hence

$$
\left|\left(x^{*}, x\right)_{\sigma}\right| \leq K\left|x^{*}\right||x|, x \in X, x^{*} \in X^{*}
$$

Condii: ons (iii) and (iv) are very nearly sufficient to ensure that $T$ is a spectral operator. In reflexive spaces they are sufficient. In general though there are operators satisfying (iii) and (iv) with

$$
\nu(\lambda)=M(\lambda)=1
$$

and not possessing a resolution of the identity. A final condition which in the case of a weakly complete space $X$ makes the set of (iii), (iv), (v) sufficient for the existence of a resolution of the identity is the following. Let $M_{\lambda}, N_{\lambda}$ be zeros and the range of $(T-\lambda)^{\nu(\lambda)}$, respectively. The condition is: 
(v) For every $\lambda$ in a set dense in $[0,1], M_{\lambda}+N_{\lambda}$ is dense in $X$.

In case $\nu(\lambda)=1$, the condition (iv) may be stated in the equivalent form:

(iv $)^{\prime} \quad \underset{\substack{0 \\ 0<\mu<1}}{\text { l.u.b. }} \int_{0}^{1}\left|x^{*}\{T(\lambda+i \mu)-T(\lambda-i \mu)\} x\right| d \lambda<\infty$.

Unless $\nu(\lambda)=1$ the condition (iv $)^{\prime}$ is more restrictive than (iv). However there is a condition analogous to (iv $)^{\prime}$ which is equivalent to (iv). It may be stated in terms of a decomposition of the resolvent. It turns out that for a spectral $T$ there are two operators $U(\xi)$ and $V(\xi)$ such that

$$
T(\xi)=U(\xi)+V(\xi)
$$

and such that $x^{*} V(\xi) x$ is the derivative of a single valued analytic function at every point $\xi$ where $x^{*} T(\xi) x$ is analytic, and $U(\xi)$ satisfies the condition (iv $)^{\prime}$. The condition (iv) may be replaced by:

(iv)" The resolvent $T(\xi)$ has a decomposition as described above.

In any one of the following situations the conditions (iii) and (iv) (or (iv $)^{\prime}$ or (iv $)^{\prime \prime}$ ) are sufficient for the existence of a resolution of the identity since in these cases ( $\mathrm{v}$ ) will automatically be satisfied:

(a) The union of the resolvent set and the continuous spectrum is dense on $[0,1]$.

(b) There is no interval of positive length consisting entirely of points in the point spectrum of the adjoint.

(c) $X$ is reflexive.

(d) $T$ is completely continuous.

Let $d(\xi)$ be the distance from $\xi$ to the spectrum $\sigma(T)$; then a condition more restrictive than (iii) is

(iii) ${ }^{\prime}$

$$
\left|d^{m}(\xi) T(\xi)\right| \leq M \text {, near } \sigma(T) .
$$

This condition is necessary and sufficient for the simplification of (ii) to

$$
f(T)=\sum_{n=0}^{m-1} \int_{\sigma(T)} \frac{f^{(n)}(\lambda)}{n !}(T-\lambda)^{n} d E_{\lambda} .
$$

Thus, in a weakly complete space, (iii) $)^{\prime}$ (iv), ( $\mathrm{v}$ ) imply that $T$ is a spectral 
operator satisfying (ii $)^{\prime}$. In a reflexive space, (iii $)^{\prime}$ and (iv) are equivalent to the statement that $T$ is a spectral operator satisfying (ii $)^{\prime}$.

In case $X$ is not weakly complete, the above statements remain valid providing the notion of the resolution of the identity is weakened in the following manner. Instead of requiring that $E_{\sigma}$ be defined for all Borel sets, we demand that it be defined and countably additive on the Boolean algebra determined by the real intervals. This enables one to define the integral occurring in (ii)' Thus in this extended sense we may say that for an arbitrary complex Banach space the conditions ( $\mathrm{iii})^{\prime}$, (iv), ( v) imply that $T$ is a spectral operator satisfying (ii)'.

Although this is the second in a series of articles on spectral theory, not much knowledge of the contents of the first [1] paper is assumed or used. We collect here the terminology, notation, and results from that paper that are used in the present one. An admissible domain is an open set bounded by a finite number of rectifiable Jordan curves. It is called a $T$-admissible domain in case its boundary is contained in the resolvent set $\rho(T)$ of $T$. The class of complex valued functions analytic and single valued on some $T$-admissible domain containing the spectrum $\sigma(T)$ is denoted by $F(T)$ or $F(\sigma(T))$. For $f \in F(T)$, the operator $f(T)$ is defined by the formula

$$
f(T)=\frac{1}{2 \pi i} \int_{C} f(\lambda) T(\lambda) d \lambda,
$$

where $C$ is the boundary of some $T$-admissible domain containing the spectrum of $T$. The mapping, given by the above formula, of the algebra of analytic functions into an algebra of operators is a homomorphism which assigns the operators $l, T$ to the functions $l, \lambda$, respectively.

\section{Operators with nondense spectra and preliminary lemmas}

In this section we consider an operator $T$ whose spectrum $\sigma(T)$ is nondense in the complex plane. Two conditions concerning the singularities of the analytic function $(\xi-T)^{-1} x$ are introduced (these are 1.7 and 1.14 below). As we show later, these are necessary conditions for the existence of a resolution of the identity regardless of the operator $T$ or the character of the space $X$. The main purpose of $\S 1$ is to show how near these two conditions come to being sufficient. Later, in $\S 2$, we shall determine the meaning of these two conditions in terms of the rate of growth and the mean rate of growth of the resolvent $T(\xi)$ for $\xi$ near the spectrum. The basic assumption for $\S 1$ is then:

1.1. Assumption. The spectrum $\sigma(T)$ of $T$ is nondense in the complex 
plane.

This means that the resolvent set $\rho(T)$ of $T$ is dense in the plane. The chief purpose of this assumption is to prove the following lemma which asserts that the analytic function $(\xi-T)^{-1} x$ is single valued; if this fact is already known then Assumption 1.1 may easily, in most of what follows, be discarded. Since 1.1 is the underlying assumption for practically all of $\S 1$, it will not be explicitly stated in the lemmas to follow. The other assumptions 1.7 and 1.14 , and others in $\$ 2$, will however be indicated parenthetically when they are used.

1.2. LEмMA. For each $x \in X$ the analytic function $T(\xi) x$ defined on $\rho(T)$ has a unique maximal single valued analytic extension.

Let $f, g$ be two vector-valued analytic functions defined on open sets $D(f)$, $D(g)$, respectively. We suppose that $D(f) D(g) \supset \rho(T)$ and that

$$
f(\xi)=T(\xi) x=g(\xi) \text { for } \xi \in \rho(T) .
$$

Let $\xi_{0} \in D(f) D(g)$. By 1.1, there is a sequence of points $\xi_{n} \in \rho(T)$ with $\xi_{n} \rightarrow \xi_{0}$, and so $f\left(\xi_{0}\right)=g\left(\xi_{0}\right)$. Thus, if $\rho(x)$ is the union of all open sets containing $\rho(T)$ upon which $T(\xi) x$ has an analytic extension, we have uniquely defined upon $\rho(x)$ an analytic extension of $T(\xi) x$.

1.3. Definitions. By $x(\xi)$ we shall mean the unique maximal single valued analytic extension of $T(\xi) x$ whose existence is established in 1.2. The symbol $\rho(x)$ will be used for the domain of definition of $x(\xi)$, and the symbol $\sigma(x)$ will be used for the set of singularities of $x(\xi)$. Thus $\sigma(x)$ is the complement of $\rho(x)$, and $\rho(x) \supset \rho(T), \sigma(x) \subset \sigma(T)$.

1.4. DEFinition. By $[x]$ we shall mean the smallest closed linear manifold containing all of the vectors $T(\xi) x, \xi \in \rho(T)$.

1.5. Lemma. For every $x \in X$ we have:

1.5.1. $x \in[x]$;

1.5.2. $f(T)[x] \subset[x], f \in F(\sigma(T))$;

1.5.3. $x(\xi) \in[x], \xi \in \rho(x)$;

1.5.4. $[y] \subset[x], y \in[x]$.

Let $C$ be a large circle such that

$$
x=\frac{1}{2 \pi i} \quad \int_{C} T(\xi) x d \xi \in[x] ;
$$


this proves 1.5.1. Let $y \in[x]$ and $f \in F(\sigma(T))$. Since $y$ may be approximated by sums of the form $\sum \alpha_{j} T\left(\xi_{j}\right) x, f(T) y$ may be approximated by sums of the form

$$
\sum \alpha_{j} T\left(\xi_{j}\right) f(T) x=\sum \alpha_{j} \frac{1}{2 \pi i} \int_{\Gamma} \frac{f(\xi) T(\xi) x}{\xi_{j}-\xi} d \xi
$$

where $\Gamma$ is chosen, in the domain of regularity of $f$, to include $\sigma(T)$ and exclude the points $\xi_{j}$. Thus $f(T) y \in[x]$, and 1.5.2 is proved. Next let $\xi_{0} \in \rho(x)$ and, using (1.1), choose a sequence $\xi_{n} \in \rho(T)$ with $\xi_{n} \longrightarrow \xi_{0}$. Thus

$$
T\left(\xi_{n}\right) x \longrightarrow x\left(\xi_{0}\right),
$$

and since $[x]$ is closed we have $x\left(\xi_{0}\right) \in[x]$. Finally if $y \in[x]$ we have, by 1.5.2, $T(\xi) y \in[x], \xi \in \rho(T)$, and thus $[y] \subset[x]$. This completes the proof of 1.5 .

1.6. Lemma. For $x, y \in X$ we have

$$
\sigma(x+y) \subset \sigma(x) \cup \sigma(y),
$$

and for $\xi \in \rho(x) \rho(y)$ we have

$$
x(\xi)+y(\xi)=(x+y)(\xi) .
$$

On the open set $\rho(x) \rho(y)$, the function $x(\xi)+y(\xi)$ is an analytic extension of

$$
T(\xi) x+T(\xi) y=T(\xi)(x+y), \quad \xi \in \rho(T) .
$$

Thus $\rho(x+y) \supset \rho(x) \rho(y)$, and for $\xi \in \rho(x) \rho(y)$ we have, by l.2,

$$
x(\xi)+y(\xi)=(x+y)(\xi) .
$$

The second assumption which is needed in most of $\S 1$ is:

1.7. Assumption. If $\sigma$ is a closed set of complex numbers, then the set $[\sigma]$ of all vectors $x$ with $\sigma(x) \subset \sigma$ is also closed.

1.8. Lemma. (Assumption 1.7.) If $\sigma$ is a closed set of complex numbers, then $[\sigma]$ is a closed linear manifold, $T[\sigma] \subset[\sigma]$, and the spectrum of $T$ when considered as an operator in $[\sigma]$ is contained in $\sigma$.

That $[\sigma]$ is a closed linear manifold follows from 1.6 and 1.7. Since 


$$
T x(\mu)=T(\mu) T x,
$$

for $\mu$ in $\rho(T)$ we have $\rho(x) \subset \rho(T x)$ or $\sigma(T x) \subset \sigma(x)$, and thus $T[\sigma] \subset[\sigma]$. Now let $x \in[\sigma], \xi \in \sigma^{\prime}$ (the complement of $\sigma$ in the whole plane), $\xi_{n} \in \rho(T)$, and $\xi_{n} \rightarrow \xi$. Since for $\mu \in \rho(T)$ we have

$$
T(\mu) x=\left(\xi_{n}-T\right) T(\mu) T\left(\xi_{n}\right) x,
$$

it follows that $\rho(x) \subset \rho\left(T\left(\xi_{n}\right) x\right)$ and thus $T\left(\xi_{n}\right) x \in[o]$. Since

$$
T\left(\xi_{n}\right) x=x\left(\xi_{n}\right) \longrightarrow x(\xi)
$$

and $[\sigma]$ is closed, we have $x(\xi) \in[\sigma]$. Thus, since

$$
(\xi-T) x(\xi)=x,
$$

it follows that

$$
(\xi-T)[\sigma]=[\sigma] .
$$

To see that $\xi-T$ is one-to-one on $[\sigma]$, suppose that

$$
(\xi-T) y=0, y \in[\sigma] \text {. }
$$

Then

$$
y(\lambda)=\frac{y}{(\lambda-\xi)} \text { and } \sigma(y) \subset(\xi) \cap \sigma=\phi,
$$

the void set. This means that $y(\lambda)$ is analytic for all $\lambda$ and thus that $y=0$. Hence, if $\xi \in \sigma^{\prime}$ then $\xi-T$ is a one-to-one map of $[\sigma]$ into all of itself.

1.9. LemmA. (Assumption 1.7.) For every pair $\sigma_{1}, \sigma_{2}$ of disjoint closed sets, there is a constant $K\left(\sigma_{1}, \sigma_{2}\right)$ such that

$$
|x(\xi)| \leq K\left(\sigma_{1}, \sigma_{2}\right)|x|, \quad \xi \in \sigma_{1}, x \in\left[\sigma_{2}\right] .
$$

By 1.8, $\sigma_{1}$ is contained in the resolvent set of $T$ when considered as an operator in $\left[\sigma_{2}\right]$. Since $x(\xi)$ is the value of this resolvent at the point $\xi \in \sigma_{1}$ when operating on $x \in\left[\sigma_{2}\right]$, the present lemma follows from the preceeding one.

1.10. LemmA. (Assumption 1.7.) For every $x \in X$ we have $T[x] \subset[x]$, and when $T$ is regarded as an operator in the space $[x]$ it has $\sigma(x)$ for its 
spectrum and $\rho(x)$ for its resolvent set.

It was proved in 1.5.2 that $T[x] \subset[x]$. Let $\rho_{1}$ be the resolvent set of $T$ as an operator in $[x]$. Using 1.5.2 again, we readily show that $\rho(T) \subset \rho_{1}$; and since $T(\xi) x$ is analytic on $\rho_{1}$ ( since $x \in[x]$, by 1.5.1), we have $\rho_{1} \subset \rho(x)$. We shall now show that for every $y \in[x]$ we have $\rho(y) \supset \rho(x)$, which means that for every $y \in[x]$ the function $T(\xi) y$ defined for $\xi \in \rho(T)$ has an analytic extension to $\rho(x)$. Elements of the form

$$
y=\sum \alpha_{j} T\left(\xi_{j}\right) x, \xi_{j} \in \rho(T)
$$

are dense in $[x]$, and for such $y$ we have, for $\mu \in \rho(T)$,

$$
T(\mu) y=\sum \alpha_{j} T\left(\xi_{j}\right) T(\mu) x .
$$

Thus $T(\mu) y$ has the analytic extension $\sum \alpha_{j} T\left(\xi_{j}\right) x(\mu), \mu \in \rho(x)$, and so, for $y$ of the form $(*)$, we have $\rho(y) \supset \rho(x), \sigma(y) \subset \sigma(x)$. Let $y \in[x]$, and let $y_{n}$ be a sequence of vectors of the form $\left(^{*}\right)$ with $y_{n} \rightarrow y$. Since $y_{n}-y_{m}$ has the form $(*)$, we have $\sigma\left(y_{n}-y_{m}\right) \subset \sigma(x)$. Let $N$ be a neighborhood whose closure $\bar{N} \subset \rho(x)$, so that $\bar{N}$ and $\sigma(x)$ are closed disjoint sets. By 1.9, then,

$$
\left|y_{n}(\xi)-y_{m}(\xi)\right|=\left|\left(y_{n}-y_{m}\right)(\xi)\right| \leq K(\bar{N}, \sigma(x))\left|y_{n}-y_{m}\right| \longrightarrow 0
$$

uniformly for $\xi \in N$. The function

$$
f(\xi)=\lim _{n} y_{n}(\xi)
$$

is analytic on $N$, and for every $\xi \in \rho(T) N$ we have

$$
f(\xi)=\lim _{n} y_{n}(\xi)=\lim _{n}(\xi-T)^{-1} y_{n}=(\xi-T)^{-1} y \text {. }
$$

Hence $f(\xi)=y(\xi), \xi \in N$, and $\rho(y) \supset \rho(x)$. Finally we let $\xi_{0} \in \rho(x)$ and show that $\xi_{0}-T$ is a one-to-one map of $[x]$ into all of itself. Let $y \in[x]$; then since $\xi_{0} \in \rho(x) \subset \rho(y)$ we have, by 1.5 .3 and 1.5.4, $y\left(\xi_{0}\right) \in[y] \subset[x]$. Since

$$
(\xi-T) y(\xi)=y
$$

for $\xi \in \rho(T)$, this same equation must hold for $\xi \in \rho(y)$; in particular,

$$
\left(\xi_{0}-T\right) y\left(\xi_{0}\right)=y .
$$


Thus $\left(\xi_{0}-T\right)[x]=[x]$. To see that $\xi_{0}-T$ is a one-to-one map on $[x]$, let $y \in[x]$ and $\left(\xi_{0}-T\right) y=0$. For large $\xi$, we have the expansion

$$
T(\xi)=\sum_{n=0}^{\infty} \frac{\left(T-\xi_{0}\right)^{n}}{\left(\xi-\xi_{0}\right)^{n+1}} ;
$$

hence $T(\xi) y=y /\left(\xi-\xi_{0}\right)$. Thus if $y \neq 0$ we have $\sigma(y)$ consisting of the single point $\xi_{0} \in \rho(x) \subset \rho(y)$, a contradiction since $\rho(y)$ and $\sigma(y)$ are disjoint. Thus it has been proved that for every $\xi \in \rho(x)$ the operator $\xi-T$ is a one-toone map of $[x]$ into all of itself, and hence $\rho(x) \subset \rho_{1} \subset \rho(x)$.

1.11. Lemma. (Assumption 1.7.) If $y \in[x]$ then $\sigma(y) \subset \sigma(x)$.

This was proved (in the form $\rho(y) \supset \rho(x)$ ) during the course of the proof of Lemma 1.10.

1.12. Lemma. (Assumption 1.7.) The set $\sigma(x)$ is void if and only if $x=0$.

If $x=0$ it follows from Definition 1.3 that $\sigma(x)$ is void. Conversely, if $\sigma(x)$ is void then by (7) the spectrum of $T$ as an operator in the space $[x]$ is void. This, according to Taylor's result [3], implies that $[x]$ consists of the zero vector alone. Hence $x=0$.

1.13. Lemma. (Assumption 1.7.) Let $\sigma$ be a set of complex numbers, and $\sigma^{\prime}$ its complement. If $x+y=x_{1}+y_{1}$, where $\sigma(x), \sigma\left(x_{1}\right) \subset \sigma$ and $\sigma(y), \sigma\left(y_{1}\right) \subset$ $\sigma^{\prime}$, then $x=x_{1}, y=y_{1}$.

The sets

$$
\sigma_{1}=\sigma(x) \cup \sigma\left(x_{1}\right), \sigma_{2}=\sigma(y) \cup \sigma\left(y_{1}\right)
$$

are bounded, closed, and disjoint. Since, by 1.6, $\sigma(x+y) \subset \sigma_{1} \cup \sigma_{2}$, there is an admissible contour $C$ containing $\sigma_{1}$ and excluding $\sigma_{2}$ which lies in $\rho(x+y)$. Thus

$$
\frac{1}{2 \pi i} \int_{C}(x+y)(\xi) d \xi=\frac{1}{2 \pi i} \int_{C} x(\xi) d \xi+\frac{1}{2 \pi i} \int_{C} y(\xi) d \xi
$$

Since $y(\xi)$ is regular in the closed domain bounded by $C$, the second integral on the right side of the above equality is zero. Since $\sigma(x)$ is contained within the domain bounded by $C$ we see, from 1.10, that the first integral on the right of the above equality is equal to $x$. Hence 


$$
\frac{1}{2 \pi i} \int_{C}(x+y)(\xi) d \xi=x,
$$

and similarly

$$
\frac{1}{2 \pi i} \int_{C}\left(x_{1}+y_{1}\right)(\xi) d \xi=x_{1}
$$

Thus $x=x_{1}, y=y_{1}$.

In most of what follows we shall need besides Assumption 1.7 the following:

1.14. Assumption. There is a constant $K$, depending only upon $T$, such that for every pair $x, y$ of vectors with $\sigma(x), \sigma(y)$ disjoint we have

$$
|x| \leq K|x+y|
$$

1.15. DEFINITION. By $s_{1}$ we shall mean the family of all sets $\sigma$ with the property that vectors of the form $x+y$ with $\sigma(x) \subset \sigma, \sigma(y) \subset \sigma^{\prime}$ are dense in $X$. Clearly, if $\sigma \in s_{1}$ then the complement $\sigma^{\prime} \in s_{1}$.

1.16. LemmA. (Assumptions 1.7, 1.14.) For $\sigma \in s_{1}$ there is one and only one bounded projection $E_{\sigma}$ on $X$ with the properties $E_{\sigma} x=x$ if $\sigma(x) \subset \sigma$; $E_{\sigma} x=0$ if $\sigma(x) \subset \sigma^{\prime}$. This projection has the further properties that

$$
E_{\sigma}+E_{\sigma^{\prime}}=I, E_{\sigma} E_{\sigma^{\prime}}=0,\left|E_{\sigma}\right| \leq K
$$

Vectors of the form $z=x+y$ with $\sigma(x) \subset \sigma, \sigma(y) \subset \sigma^{\prime}$ are dense in $X$. In view of 1.13 it is permissible to define, on this dense set, $E_{\sigma} z=x$. From 1.14 it follows that $\left|E_{\sigma} z\right| \leq K|z|$. Now if

$$
z_{1}=x_{1}+y_{1} \text { with } \sigma\left(x_{1}\right) \subset \sigma, \sigma\left(y_{1}\right) \subset \sigma^{\prime},
$$

then

$$
z+z_{1}=x+x_{1}+y+y_{1}
$$

and, by 1.6, $\sigma\left(x+x_{1}\right) \subset \sigma, \sigma\left(y+y_{1}\right) \subset \sigma^{\prime}$. Thus

$$
E_{\sigma}\left(z+z_{1}\right)=E_{\sigma} z+E_{\sigma} z_{1}
$$

and $E_{\sigma}$ is additive and continuous on a dense linear set. Thus $E_{\sigma} z$ is uniquely 
defined for $z \in X$ by the requirements that $E_{\sigma} z$ is continuous in $z$. For elements $z$ of the original dense set we have

$$
E_{\sigma}^{2} z=E_{\sigma} x=x=E_{\sigma} z \text {, where } z=x+y, \sigma(x) \subset \sigma, \sigma(y) \subset \sigma^{\prime} .
$$

Thus $E_{\sigma}^{2}=E_{\sigma}$. It is also clear that

$$
E_{\sigma} E_{\sigma^{\prime}}=0 \text { and } E_{\sigma}+E_{\sigma^{\prime}}=I .
$$

If $A_{\sigma}$ is another bounded projection with the properties

$$
A_{\sigma} x=x \text { if } \sigma(x) \subset \sigma \text { and } A_{\sigma} x=0 \text { if } \sigma(x) \subset \sigma^{\prime} \text {, }
$$

then for $z=x+y$, where $\sigma(x) \subset \sigma, \sigma(y) \subset \sigma^{\prime}$ we have $A_{\sigma} z=x=E_{\sigma} z$, and hence $A_{\sigma} z=E_{\sigma} z$ for every $z \in X$.

1.17. Lemma. (Assumptions 1.7, 1.14.) If $\sigma \in s_{1}$ and $f \in F(\sigma(T))$, then $f(T) E_{\sigma}=E_{\sigma} f(T)$.

Let $z=x+y, \sigma(x) \subset \sigma, \sigma(y) \subset \sigma^{\prime}$. Then

$$
f(T) z=f(T) x+f(T) y .
$$

By 1.5.1 and 1.5.2, $f(T) x \in[x]$; and by 1.11, $\sigma(f(T) x) \subset \sigma(x) \subset \sigma$. Similarly, $\sigma(f(T) y) \subset \sigma(y) \subset \sigma^{\prime}$. So

$$
E_{\sigma} f(T) z=f(T) x=f(T) E_{\sigma} z
$$

Since the vectors $z$ are dense, the lemma is proved.

1.18. LemmA. (Assumptions 1.7, 1.14.) We have $\sigma\left(E_{\sigma} x\right) \subset \sigma(x), \sigma \in s_{1}$, $x \in X$.

We have, by 1.17,

$$
T(\xi) E_{\sigma} x=E_{\sigma} T(\xi) x, \xi \in \rho(T),
$$

and hence the analytic function $T(\xi) E_{\sigma} x$ has the analytic extension $E_{\sigma} x(\xi)$ for $\xi \in \rho(x)$. Thus $\rho\left(E_{\sigma} x\right) \supset \rho(x)$ and $\sigma\left(E_{\sigma} x\right) \subset \sigma(x)$.

1.19. Definition. For $\sigma \in s_{1}$, define $X_{\sigma}=E_{\sigma} X$.

1.20. Definition. If $M$ is a closed linear manifold in $X$ for which $T M \subset M$, we use the symbol $\sigma(M)$ for the spectrum of $T$ when considered as an operator in $M$, and the symbol $\rho(M)$ for the resolvent set of $T$ as an operator in $M$. 
1.21. Theоrem. (Assumptions 1.7, 1.14.) If $\sigma \in s_{1}$, then $T X_{\sigma} \subset X_{\sigma}$ and $\sigma\left(X_{\sigma}\right) \subset \bar{\sigma}$, where $\bar{\sigma}$ is the closure of $\sigma$.

It follows from 1.17 that $T X_{\sigma} \subset X_{\sigma}$. Let $\xi \notin \varnothing \bar{\sigma}$. We shall first show that $\xi-T$ is one-to-one on $X_{\sigma}$. If $x \in X_{\sigma},(\xi-T) x=0$, then $x(\lambda)=x /(\lambda-\xi)$ since for all large $\lambda$ we have

$$
T(\lambda)=\sum_{n=0}^{\infty} \frac{(T-\xi)^{n}}{(\lambda-\xi)^{n+1}} .
$$

Since $x \in X_{\sigma}$, we have $x=E_{\sigma} x$; and since $x(\lambda)$ is everywhere regular except possibly at the point $\xi \in \sigma^{\prime}$, we have $\sigma(x) \subset \sigma^{\prime}$, from which it follows that $E_{\sigma} x=0$. Thus $\xi-T$ is one-to-one on $X_{\sigma}$. We next show that $(\xi-T) X_{\sigma}=X_{\sigma}$. Let

$$
x \in X_{\sigma} \text { and } x_{n}+y_{n} \longrightarrow x, \sigma\left(x_{n}\right) \subset \sigma, \sigma\left(y_{n}\right) \subset \sigma^{\prime} .
$$

Then

$$
x=E_{\sigma} x=\lim _{n} E_{\sigma}\left(x_{n}+y_{n}\right)=\lim _{n} E_{\sigma} x_{n}=\lim _{n} x_{n} .
$$

Let $y_{n}=x_{n}(\xi)$, so that

$$
y_{n}-y_{m}=x_{n}(\xi)-x_{m}(\xi)=\left(x_{n}-x_{m}\right)(\xi),
$$

and hence, by 1.9 ,

$$
\left|y_{n}-y_{m}\right| \leq K_{1}\left|x_{n}-x_{m}\right| \rightarrow 0 \text {. }
$$

Let $y=\lim _{n} y_{n}$, so that

$$
x=\lim _{n} x_{n}=\lim (\xi-T) y_{n}=(\xi-T) y .
$$

It remains to be shown that $y \in X_{\sigma}$. Since $\xi \in \rho\left(x_{n}\right)$, we see from 1.5.3 that

$$
y_{n}=x_{n}(\xi) \in\left[x_{n}\right],
$$

and thus 1.11 gives $\sigma\left(y_{n}\right) \subset \sigma\left(x_{n}\right) \subset \sigma$. Thus $y_{n}=E_{\sigma} y_{n} \in X_{\sigma}$ and $y \in X_{\sigma}$. We have shown that if $\xi \notin \bar{\sigma}$ then $\xi-T$ is a one-to-one map of $X_{\sigma}$ into all of itself; that is, $\sigma\left(X_{\sigma}\right) \subset \bar{\sigma}$.

1.22. LemmA. (Assumptions 1.7, 1.14.) If $\sigma \in s_{1}$, then $\sigma\left(E_{\sigma} x\right) \subset \bar{\sigma} \sigma(x)$ 
for every $x \in X$.

In view of 1.18 it will suffice to show that $\sigma\left(E_{\sigma} x\right) \subset \bar{\sigma}$. From 1.21 it follows that $\rho\left(E_{\sigma} x\right) \supset \bar{\sigma}^{\prime}$, and thus $\sigma\left(E_{\sigma} x\right) \subset \bar{\sigma}$.

1.23. Definition. The symbol $s_{2}$ will be used for the family of all sets $\sigma$ having the following property. For every $x \in X$ and every $\epsilon>0$ there are vectors $x_{1}, x_{1}^{\prime}$ with $\sigma\left(x_{1}\right) \subset \sigma(x) \sigma, \sigma\left(x_{1}^{\prime}\right) \subset \sigma(x) \sigma^{\prime}$ and $\left|x_{1}+x_{1}^{\prime}-x\right|<\epsilon$.

1.24. Lemma. The family $s_{2}$ is a Boolean algebra and $s_{2} \subset s_{1}$.

That $s_{2} \subset s_{1}$ is clear from the definition of these classes. Let $\sigma_{1}, \sigma_{2} \in s_{2}$, $x \in X$ and $\epsilon>0$. We then have

$$
\begin{aligned}
& x=x_{1}+x_{1}^{\prime}+u_{1}, \sigma\left(x_{1}\right) \subset \sigma(x) \sigma_{1}, \sigma\left(x_{1}^{\prime}\right) \subset \sigma(x) \sigma_{1}^{\prime},\left|u_{1}\right|<\epsilon / 2 ; \\
& x_{1}^{\prime}=x_{2}+x_{2}^{\prime}+u_{2}, \sigma\left(x_{2}\right) \subset \sigma\left(x_{1}^{\prime}\right) \sigma_{2}, \sigma\left(x_{2}^{\prime}\right) \subset \sigma\left(x_{1}^{\prime}\right) \sigma_{2}^{\prime},\left|u_{2}\right|<\epsilon / 2 ; \\
& x=x_{1}+x_{2}+x_{2}^{\prime}+u_{1}+u_{2} .
\end{aligned}
$$

Using 1.6 we see that

$$
\begin{aligned}
\sigma\left(x_{1}+x_{2}\right) \subset \sigma\left(x_{1}\right) \cup \sigma\left(x_{2}\right) & \subset\left(\sigma(x) \sigma_{1}\right) \cup\left(\sigma\left(x_{1}^{\prime}\right) \sigma_{2}\right) \\
& \subset\left(\sigma(x) \sigma_{1}\right) \cup\left(\sigma(x) \sigma_{2}\right) \\
& =\sigma(x)\left(\sigma_{1} \cup \sigma_{2}\right),
\end{aligned}
$$

and

$$
\sigma\left(x_{2}^{\prime}\right) \subset \sigma\left(x_{1}^{\prime}\right) \sigma_{2}^{\prime} \subset \sigma(x) \sigma_{1}^{\prime} \sigma_{2}^{\prime}=\sigma(x)\left(\sigma_{1} \cup \sigma_{2}\right)^{\prime} .
$$

Thus $\sigma_{1} \cup \sigma_{2} \dot{\epsilon} s_{2}$. It is clear from 1.23 that $s_{2}$ is closed under complementation and that the void set and the whole plane are in $s_{2}$. Thus $s_{2}$ is closed under crosscut; that is, $\sigma_{1} \sigma_{2} \in s_{2}$ if $\sigma_{1}, \sigma_{2} \in s_{2}$, and $s_{2}$ is a Boolean algebra.

1.25. Theоrem. (Assumptions 1.7, 1.14.) On the Boolean algebra $s_{2}$ the projections $E_{\sigma}$ have the following properties:

$$
\begin{aligned}
& E_{\sigma_{1}} \cup E_{\sigma_{2}}=E_{\sigma_{1}} \cup \sigma_{2}, E_{\sigma_{1}} E_{\sigma_{2}}=E_{\sigma_{1} \sigma_{2}}, E_{\sigma}^{\prime}=E_{\sigma^{\prime}} ; \\
& E_{\sigma(T)}=1, E_{\phi}=0, \text { where } \phi \text { is the void set. }
\end{aligned}
$$


If the projections $E_{\sigma}, \sigma \in s_{2}$ are ordered in the usual fashion (that is, $E_{\sigma_{1}} \subset E_{\sigma_{2}}$ means $E_{\sigma_{2}} E_{\sigma_{1}}=E_{\sigma_{1}}$ or equivalently $\left.X_{\sigma_{1}} \subset X_{\sigma_{2}}\right)$ then, by definition, $E_{\sigma_{1}} \cup E_{\sigma_{2}}$ is the smallest projection containing $E_{\sigma_{1}}$ and $E_{\sigma_{2}}$. It may be given by the formula

$$
E_{\sigma_{1}} \cup E_{\sigma_{2}}=E_{\sigma_{1}}+E_{\sigma_{2}}-E_{\sigma_{1}} E_{\sigma_{2}}
$$

This formula is readily derived from the relation

$$
E_{\sigma_{1}} E_{\sigma_{2}}=E_{\sigma_{2}} E_{\sigma_{1}}
$$

which of course will be established as soon as we have shown that

$$
E_{\sigma_{1}} E_{\sigma_{2}}=E_{\sigma_{1} \sigma_{2}}
$$

Now let $x \in X, \epsilon>0, \sigma_{1}, \sigma_{2} \in s_{2}$. We have

$$
x=x_{1}+x_{1}^{\prime}+u, x_{1}^{\prime}=x_{2}+x_{2}^{\prime}+v, x_{1}=y_{2}+y_{2}^{\prime}+w,
$$

where $|u|,|v|,|w|<\epsilon$, and

$$
\begin{aligned}
& \sigma\left(x_{1}\right) \subset \sigma(x) \sigma_{1}, \quad \sigma\left(x_{1}^{\prime}\right) \subset \sigma(x) \sigma_{1}^{\prime}, \\
& \sigma\left(x_{2}\right) \subset \sigma\left(x_{1}^{\prime}\right) \sigma_{2} \subset \sigma(x) \sigma_{1}^{\prime} \sigma_{2} \subset\left(\sigma_{1} \sigma_{2}\right)^{\prime}, \\
& \sigma\left(x_{2}^{\prime}\right) \subset \sigma\left(x_{1}^{\prime}\right) \sigma_{2}^{\prime} \subset \sigma(x) \sigma_{1}^{\prime} \sigma_{2}^{\prime} \subset\left(\sigma_{1} \cup \sigma_{2}\right)^{\prime} \subset\left(\sigma_{1} \sigma_{2}\right)^{\prime}, \\
& \sigma\left(y_{2}\right) \subset \sigma\left(x_{1}\right) \sigma_{2} \subset \sigma(x) \sigma_{1} \sigma_{2}, \\
& \sigma\left(y_{2}^{\prime}\right) \subset \sigma\left(x_{1}\right) \sigma_{2}^{\prime} \subset \sigma(x) \sigma_{1} \sigma_{2}^{\prime} \subset\left(\sigma_{1} \sigma_{2}\right)^{\prime} \sigma_{1} .
\end{aligned}
$$

Place $z=y_{2}+y_{2}^{\prime}+x_{2}+x_{2}^{\prime}, \quad y=u+v+w$, so that $x=z+y$ and $|y|<3 \epsilon$. Remembering that $E_{\sigma} x=x$ for every $x$ with $\sigma(x) \subset \sigma$ and $E_{\sigma} x=0$ if $\sigma(x) \subset \sigma^{\prime}$, we see from the above inclusion relations that

$$
\begin{aligned}
& E_{\sigma_{1}} z=y_{2}+y_{2}^{\prime}, E_{\sigma_{2}} z=y_{2}+x_{2}, \\
& E_{\sigma_{2}} E_{\sigma_{1}} z=E_{\sigma_{1}} E_{\sigma_{2}} z=E_{\sigma_{1} \sigma_{2}} z=y_{2}, \\
& \left(E_{\sigma_{1}}+E_{\sigma_{2}}-E_{\sigma_{1}} E_{\sigma_{2}}\right) z=y_{2}+y_{2}^{\prime}+x_{2}=E_{\sigma_{1}} \cup \sigma_{2} z .
\end{aligned}
$$

Hence 


$$
\left|\left(E_{\sigma_{1}} E_{\sigma_{2}}-E_{\sigma_{1} \sigma_{2}}\right) x\right|=\left|\left(E_{\sigma_{1}} E_{\sigma_{2}}-E_{\sigma_{1} \sigma_{2}}\right) y\right| \leq 3 K(K+1) \epsilon .
$$

Since $\epsilon$ is independent of $x$, we have

$$
E_{\sigma_{1}} E_{\sigma_{2}}=E_{\sigma_{1} \sigma_{2}}=E_{\sigma_{2}} E_{\sigma_{1}} \text {. }
$$

Also,

$$
\left|\left(\left(E_{\sigma_{1}} \cup E_{\sigma_{2}}\right)-E_{\sigma_{1}} \cup \sigma_{2}\right) x\right| \leq 4 K|y|<12 K \epsilon,
$$

so that $E_{\sigma_{1}} \cup E_{\sigma_{2}}=E_{\sigma_{1}} \cup \sigma_{2}$. The remaining conclusions have been proved in 1.16 .

1.26. DEFINITION. (Assumptions $1.7,1.14$.) The symbol $s_{3}$ will be used for those sets $\sigma \in_{s_{1}}$ for which there exist closed sets $\mu_{n}, \nu_{n} \in s_{2}$ with $\nu_{n} \subset \sigma, \mu_{n} \subset \sigma^{\prime}, n=1,2, \cdots$ and

$$
x=\lim _{n}\left(E_{\nu_{n}}+E_{\mu_{n}}\right) x, x \in X .
$$

1.27. Lemma. (Assumptions $1.7,1.14$.) The family $s_{3}$ is a Boolean algebra and $s_{3} \subset s_{2}$.

If $\sigma \in s_{3}$ and $\mu_{n}, \nu_{n}$ are as in 1.26 , then by 1.22 we have

$$
\sigma\left(E_{\nu_{n}} x\right) \subset \nu_{n} \sigma(x) \subset \sigma \sigma(x), \sigma\left(E_{\mu_{n}} x\right) \subset \mu_{n} \sigma(x) \subset \sigma^{\prime} \sigma(x),
$$

and so $\sigma \in s_{2}$; that is, $s_{3} \subset s_{2}$. It is clear that $s_{3}$ is closed under complementation; hence, in order to show that $s_{3}$ is a Boolean algebra, it will suffice to show that it is closed under the operation of forming unions. Let $\sigma_{1}, \sigma_{2} \in s_{3}$ and $\nu(i, n), \mu(i, n),(i=1,2 ; n=1,2, \ldots)$ be closed $s_{2}$ sets with $\nu(i, n) \subset$ $\sigma_{i}, \mu(i, n) \subset \sigma_{i}^{\prime}(i=1,2)$ and

$$
x=\lim _{n}\left(E_{\nu(i, n)}+E_{\mu(i, n)}\right) x
$$

Then

$$
x=E_{\nu(1, n)} x+E_{\mu(1, n)} x+u_{n}, \text { and } u_{n} \rightarrow 0 \text {. }
$$

Thus

$$
E_{\sigma_{1}^{\prime}} x=E_{\mu(1, n)} x+E_{\sigma_{1}^{\prime}} u_{n} \text { and } E_{\mu(1, n)} x \rightarrow E_{\sigma_{1}^{\prime}} x
$$


This last fact shows that the sequence $v_{n}$ defined by the next equation has the property that $v_{n} \rightarrow 0$ :

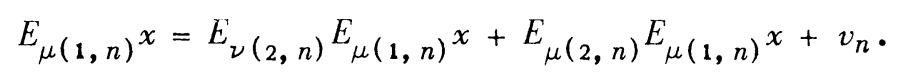

Upon substituting the above expression for $E_{\mu(1, n)^{x}}$ into the formula defining $u_{n}$, we see by using 1.25 that

$$
x=E_{\nu_{n}} x+E_{\mu_{n}} x+u_{n}+v_{n},
$$

where

$$
\nu_{n}=\nu(1, n) \cup \nu(2, n) \mu(1, n) \text { and } \mu_{n}=\mu(2, n) \mu(1, n) .
$$

Since $\nu_{n}, \mu_{n}$ are closed $s_{2}$ sets (by 1.24) with $\nu_{n} \subset \sigma_{1} \cup \sigma_{2}$, and $\mu_{n} \subset \sigma_{1}^{\prime} \sigma_{2}^{\prime}=$ $\left(\sigma_{1} \cup \sigma_{2}\right)^{\prime}$, it follows that $\sigma_{1} \cup \sigma_{2}$ is an $s_{3}$ set, and the lemma is established.

1.28. Lemma. (Assumptions 1.7, 1.14.) Let $\sigma \in s_{3}, x \in X, \epsilon>0$. Then there are sets $\mu, \nu \in s_{2}$ with $\mu$ open, $\nu$ closed, $\mu \supset \sigma \supset \nu$, and such that

$$
\begin{aligned}
& \left|E_{\omega} x\right|<\epsilon, \omega \subset \mu-\nu, \omega \in s_{2} ; \\
& \left|E_{\sigma} x-E_{\sigma_{1}} x\right|<\epsilon, \mu \supset \sigma_{1} \supset \nu, \sigma_{1} \in s_{2} .
\end{aligned}
$$

Since $\sigma$ is an $s_{3}$ set, there are closed sets $\nu, \mu^{\prime} \in s_{2}$ with

$$
\nu \subset \sigma, \mu^{\prime} \subset \sigma^{\prime}, x=E_{\nu} x+E_{\mu} x+u \text {, and }|u|<\epsilon .
$$

Then

$$
\left|E_{\mu} x-E_{\nu} x\right|=\left|E_{\mu} u\right|<K \epsilon \text {. }
$$

Let $\omega \subset \mu-\nu, \omega \in s_{2}$. Then

$$
\left|E_{\omega} x\right|=\left|E_{\omega} E_{\mu-\nu} x\right|=\left|E_{\omega}\left(E_{\mu}-E_{\nu}\right) x\right|<K^{2} \epsilon ;
$$

this proves the first conclusion. Now

$$
E_{\sigma}-E_{\sigma_{1}}=E_{\sigma-\sigma \sigma_{1}}+E_{\sigma \sigma_{1}}-E_{\sigma_{1}-\sigma \sigma_{1}}-E_{\sigma \sigma_{1}}=E_{\sigma-\sigma \sigma_{1}}-E_{\sigma_{1}-\sigma \sigma_{1}},
$$

and since $\sigma-\sigma \sigma_{1} \subset \mu-\nu, \sigma_{1}-\sigma \sigma_{1} \subset \mu-\nu$, the second conclusion follows from the first conclusion. 
1.29. LemmA. (Assumption 1.7, 1.14.) If $\sigma_{m}, \sigma \in s_{3} ; \sigma_{m} \subset \sigma_{m+1}(m=$ $1,2, \cdots)$; and $\sigma_{m} \rightarrow \sigma$; then $E_{\sigma_{m}} x \rightarrow E_{\sigma} x, x \in X$.

Let $x \in X, \epsilon>0$ be arbitrary. Let $\epsilon_{n}>0$ and $\sum_{n=1}^{\infty} \epsilon_{n}<\epsilon$. Using 1.28, pick open sets $\mu_{n} \in s_{2}$ with $\mu_{n} \supset \sigma_{n}$ and

$$
\left|E_{\omega} x\right|<\epsilon_{n}, \omega \subset \mu_{n}-\sigma_{n}, \omega \in s_{2} .
$$

We shall now show that

$$
\left|E_{\omega} x\right|<\sum_{j=1}^{n} \epsilon_{j}, \omega \subset\left(\mu_{1}+\cdots+\mu_{n}\right)-\sigma_{n}, \omega \in s_{2} .
$$

The statement $\left(^{*}\right)$ is true for $n=1$. Assume that it is true for $n$ and let

$$
\omega \subset\left(\mu_{1}+\cdots+\mu_{n+1}\right)-\sigma_{n+1}, \omega \in s_{2},
$$

so that

$$
\omega=\omega\left[\left(\mu_{1}+\cdots+\mu_{n}\right)-\sigma_{n}\right]+\omega\left(\mu_{n+1}-\sigma_{n+1}\right)
$$

and $\omega=\omega_{1}+\omega_{2}$, where

$$
\omega_{1}=\omega\left[\left(\mu_{1}+\cdots+\mu_{n}\right)-\sigma_{n}\right]\left(\mu_{n+1}-\sigma_{n+1}\right)^{\prime}, \omega_{2}=\omega\left(\mu_{n+1}-\sigma_{n+1}\right) .
$$

By our induction assumption we have

$$
\left|E_{\omega_{1}} x\right|<\sum_{j=1}^{n} \epsilon_{j}
$$

and since $\omega_{2} \subset \mu_{n+1}-\sigma_{n+1}$ we have

$$
\left|E_{\omega_{2}} x\right|<\epsilon_{n+1}
$$

Since $\omega_{1}$ and $\omega_{2}$ are disjoint, it follows that

$$
\left|E_{\omega} x\right| \leq\left|E_{\omega_{1}} x\right|+\left|E_{\omega_{2}} x\right|<\sum_{j=1}^{n+1} \epsilon_{j}
$$

this proves $(*)$. Now let $\xi_{n}=\mu_{1}+\cdots+\mu_{n}$, so that $\xi_{n}$ is open, increasing with $n, \xi_{n} \supset \sigma_{n}$, and $\left|E_{\omega} x\right|<\epsilon$ for every $\omega \epsilon^{\prime} s_{2}$ with $\omega \subset \xi_{n}-\sigma_{n}$. Using 1.28, let 
$\mu, \nu \in s_{2}, \mu$ open, $\nu$ closed, $\mu \supset \sigma \supset \nu$, and $\left|E_{\sigma} x-E_{\sigma_{1}} x\right|<\epsilon$ for every $\sigma_{1} \in s_{2}$ with $\mu \supset \sigma_{1} \supset \nu$. We have

$$
\mu \supset \bigcup_{m=1}^{\infty} \xi_{m} \mu \supset \mu \bigcup_{m=1}^{\infty} \sigma_{m}=\mu \sigma \supset \sigma \supset \nu .
$$

Since $\nu$ is closed, there is an integer $k_{0}$ such that

$$
\mu \supset \underset{m=1}{\mathrm{U}} \xi_{m} \mu=\xi_{k} \mu \supset \nu,
$$

Thus

$$
\left|E_{\sigma} x-E_{\xi_{k} \mu} x\right|<\epsilon
$$

and since $\xi_{k} \mu-\sigma_{k} \subset \xi_{k}-\sigma_{k}$ we have, from (*),

$$
\left|E_{\xi_{k} \mu} x-E_{\sigma_{k}} x\right|=\left|E_{\xi_{k} \mu \cdot \sigma_{k}} x\right|<\epsilon, \quad(k=1,2, \cdots) .
$$

Hence

$$
\left|E_{\sigma} x-E_{\sigma_{k}} x\right|<\epsilon, \quad\left(k \geq k_{0}\right)
$$

this proves the lemma.

1.30. The OREM. (Assumptions $1.7,1.14$.$) For each x \in X$ the set function $E_{\sigma} x$ is countably additive on the Boolean algebra $s_{3}$.

The conclusion of the theorem means that if $\sigma, \sigma_{n} \in s_{3}, \sigma_{n} \sigma_{m}$ is void for $n \neq m, \sigma=\cup_{1}^{\infty} \sigma_{n}$, then

$$
\sum_{n=1}^{\infty} E_{\sigma_{n}} x=E_{\sigma} x .
$$

The lemma is an immediate consequence of $1.25,1.27$, and 1.29 .

1.31. Definition. By a Borel algebra of sets we shall mean a Boolean algebra of sets which is closed under the operation of taking denumerable unions. 
1.32. Definition. The smallest Borel algebra of sets containing the Boolean algebra $s_{3}$ will be called the family of sets measurable $T$ and will be denoted by $m(T)$.

In part of what follows we shall assume:

\subsection{Assumption. The space $X$ is weakly complete.}

1.34. ThEOREm. (Assumptions $1.7,1.14,1.33$.$) The function E_{e}$ defined on $s_{3}$ to the set of bounded projections on $X$ has a unique extension to $m(T)$ with the properties (the first of which ensures uniqueness):

(i) $E_{e} x$ is countably additive on $m(T), x \in X$;

(ii) $\left|E_{e}\right| \leq K, \quad e \in m(T)$;

(iii) $E_{e_{1}} E_{e_{2}}=E_{e_{1} e_{2}}, e_{1}, e_{2} \in m(T)$;

(iv) $E_{e_{1} \cup e_{2}}=E_{e_{1}} \cup E_{e_{2}}, e_{1}, e_{2} \in m(T)$;

(v) $E_{e}^{\prime}=E_{e}, E_{\sigma(T)}=I, E_{\phi}=0, e \in m(T), \phi$ void;

(vi) $f(T) E_{e}=E_{e} f(T), e \in m(T), f \in F(\sigma(T))$.

For point sets $e_{n}, e$ we mean by $e_{n} \longrightarrow e$ or $\lim _{n} e_{n}=e$ that

$$
e=\bigcap_{m=1}^{\infty} \underset{n=m}{\bigcup} e_{n}=\bigcup_{m=1}^{\infty} \bigcap_{n=m}^{\infty} e_{n},
$$

and we recall that if

$$
a_{n} \rightarrow a, b_{n} \rightarrow b
$$

then

$$
a_{n} b_{n} \longrightarrow a b, a_{n} \cup b_{n} \longrightarrow a \cup b, a_{n}^{\prime} \longrightarrow a^{\prime}
$$

We define a transfinite sequence of Boolean algebras $\beta_{0}, \beta_{1}, \ldots$ as follows: $\beta_{0}=s_{3}$ and $\beta_{a}$ consists of all $e$ such that there exists a sequence

$$
e_{n} \in \underset{\gamma<a}{U} \beta_{\gamma}
$$

with $e_{n} \rightarrow e$. Thus $m(T)=U_{\gamma<\omega} \beta_{\gamma}$, where $\omega$ is the first ordinal whose cardinal is that of a nonenumerable class. For each $x \in X$ and $x^{*} \in X^{*}$ there is, according to a well-known theorem of Hahn, a uniquely defined countably 
additive numerical set function $m\left(e, x, x^{*}\right)$ on $m(T)$ such that

$$
m\left(e, x, x^{*}\right)=x^{*} E_{e} x, e \in s_{3}=\beta_{0}, x \in X, x^{*} \in X^{*} .
$$

We first show that for every $x \in X$ and $e \in m(T)$ there is a unique vector $x_{e} \in X$ such that

$$
x^{*} x_{e}=m\left(e, x, x^{*}\right), x^{*} \in X^{*} .
$$

This is true for $e \in \beta_{0}$. Assume that it is true for $e \in U_{\gamma<\alpha} \beta_{\gamma}$ and let $e \in \beta_{\alpha}$, $e_{n} \in U_{\gamma<a} \beta_{\gamma}, e_{n} \rightarrow e$. Then

$$
x^{*} x_{e_{n}}=m\left(e_{n}, x, x^{*}\right) \longrightarrow m\left(e, x, x^{*}\right), x^{*} \in X^{*} .
$$

Since $X$ is weakly complete, there is a vector $x_{e}$ with

$$
x^{*} x_{e}=m\left(e, x, x^{*}\right), x^{*} \in X^{*} .
$$

This last equation shows that $x_{e}$ is independent of the sequence $e_{n} \rightarrow e$ and also is uniquely defined. Next consider the statements:

$$
\begin{aligned}
& \left|m\left(e, x, x^{*}\right)\right| \leq K|x|\left|x^{*}\right| ; \\
& m\left(e, x_{1}+x_{2}, x^{*}\right)=m\left(e, x_{1}, x^{*}\right)+m\left(e, x_{2}, x^{*}\right) ; \\
& m\left(e, \alpha x, x^{*}\right)=\alpha m\left(e, x, x^{*}\right), \alpha \text { scalar. }
\end{aligned}
$$

These relations hold for $e \in \beta_{0}$, and since $m\left(e, x, x^{*}\right)$ is continuous on $m(T)$ in the topology $e_{n} \longrightarrow e$ it is seen by induction that they hold for any $e \in m(T)$. They show that for fixed $e \in m(T)$ the vector $x_{e}$ is linear and continuous in $x$; that is, for $e \in m(T)$ there is a bounded linear operator $E_{e}$ on $X$ with $E_{e} x=$ $x_{e}$. Hence we have

$$
x^{*} E_{e} x=m\left(e, x, x^{*}\right),\left|E_{e}\right| \leq K, e \in m(T), x \in X, x^{*} \in X^{*} .
$$

The uniqueness of $E_{\boldsymbol{e}}$ follows from the uniqueness of $m\left(e, x, x^{*}\right)$ asserted by Hahn's theorem. That $E_{e} x$ is countably additive on $m(E)$ in the strong topology of operators and not merely in the weak topology follows from a theorem of Orlicz concerning weakly complete spaces. Banach has restated the theorem of Orlicz in a form to hold on any Banach space and it reads as follows [2]:

Orlicz-BAnach TheOrem. If all the partial sums of $\sum_{x_{n}}$ converge weakly to an element, then the series $\sum_{x_{n}}$ is unconditionally convergent. 
The countable additivity of $E_{e} x$ is a corollary. For let

$$
e_{n} e_{m}=\phi, n \neq m, e_{n} \in m(T), e=\bigcup_{n=1}^{\infty} e_{n}
$$

For every set $\pi$ of integers, let

$$
e_{\pi}=\underset{n \in \pi}{\bigcup} e_{n}
$$

Then we have the weak series convergence:

$$
\sum_{n \in \pi} E_{e_{n}} x=E_{e_{\pi}} x
$$

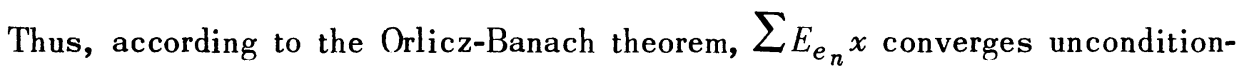
ally in the strong vector topology. The sum is, of course, $E_{e} x$ since

$$
x^{*} E_{e} x=\sum_{x^{*}} E_{e_{n}} x, x^{*} \in X^{*} .
$$

Thus we have proved statements (i) and (ii). Statement (iii) holds for $e_{1}$, $e_{2} \in \beta_{0}$. We suppose that

$$
E_{a} E_{b}=E_{a b}, a, b \in \underset{\gamma<a}{\cup} \beta_{\gamma},
$$

and let

$$
a, b_{n} \in \underset{\gamma<a}{\mathbf{U}} \beta_{\gamma} \text { with } b_{n} \rightarrow b \in \beta_{\alpha} .
$$

Then

$$
\begin{aligned}
x^{*} E_{a} E_{b} x=m\left(b, x, x^{*} E_{a}\right)=\lim _{n}\left(b_{n}, x, x^{*} E_{a}\right)=\lim _{n} x^{*} E_{a} E_{b_{n}} x \\
=\lim _{n} x^{*} E_{a b_{n}} x=\lim _{n} m\left(a b_{n}, x, x^{*}\right)=m\left(a b, x, x^{*}\right)=x^{*} E_{a b} x .
\end{aligned}
$$

Thus

$$
E_{a} E_{b}=E_{a b} \text { for } a \in \underset{\gamma<a}{\cup} \beta_{\gamma}, b \in \beta_{a} .
$$

Next choose 


$$
a_{n} \in \underset{\gamma<a}{\bigcup} \beta_{\gamma} \text { with } a_{n} \rightarrow a \in \beta_{a} .
$$

Then

$$
\begin{aligned}
x^{*} E_{a} E_{b} x & =m\left(a, E_{b} x, x^{*}\right)=\lim _{n} m\left(a_{n}, E_{b} x, x^{*}\right)=\lim _{n} x^{*} E_{a_{n}} E_{b} x \\
& =\lim _{n} x^{*} E_{a_{n} b} x=\lim _{n} m\left(a_{n} b, x, x^{*}\right)=m\left(a b, x, x^{*}\right)=x^{*} E_{a b} x .
\end{aligned}
$$

This proves (iii). Statements ( $v$ ) and (vi) are readily proved by induction, and (iv) follows from (iii) and (v).

1.35. Definition. If for each $e \in B$, the Borel sets in the complex plane, there is a bounded linear operator $E_{e}$ on $X$, then the function $E_{e}$ on $B$ to the ring of operators on $X$ is called a resolution of the identity in case $E_{e^{\prime}}=I-E_{e}$, $E_{e_{1}} E_{e_{2}}=E_{e_{1} e_{2}}$ for $e, e_{1}, e_{2} \in B$, and $x^{*} E_{e} x$ is countably additive on $B$ for every $x \in X, x^{*} \in X^{*}$.

1.36. LemmA. A resolution of the identity has the further properties

(i) $E_{e} x$ is countably additive on $B, x \in X$;

(ii) $\sup _{\boldsymbol{e} \in B}\left|E_{e}\right|<\infty$;

(iii) $E_{e_{1}} E_{e_{2}}=E_{e_{1} e_{2}}, E_{e_{1} \cup e_{2}}=E_{e_{1}} \cup E_{e_{2}}, e_{1}, e_{2} \in B$;

(iv) $E_{e}^{\prime}=E_{e}, E_{\phi}=0, E_{p}=1, e \in B, \phi$ void, $p=$ the whole plane.

Statement (i) follows from the Orlicz-Banach theorem, and (ii) from the principle of uniform boundedness. $E_{\phi}=0$ since $E_{e}$ is additive in $e$; hence $I=E_{\dot{\phi}}^{\prime}=E_{p}$. The second part of (iii) follows from the first part and (iv).

1.37. Definition. A resolution of the identity $E_{e}$ is called a resolution of the identity for the linear operator $T$ in case

$$
T E_{e}=E_{e} T \text { and } \sigma\left(E_{e} X\right) \subset \bar{e}, e \in B .
$$

1.38. LemmA. Let $X$ be weakly complete, and let $T$ be a bounded linear operator in $X$ whose spectrum is nondense. Then $T$ has a resolution of the identity if and only if it satisfies the conditions 1.7, 1.14 and:

1.39. For every complex number $\lambda$ and every $\epsilon>0$ there is an $s_{3}$ set of diameter $<\epsilon$ and containing $\lambda$ as an interior point.

Furthermore, when $T$ has a resolution of the identity $E_{e}$ it is unique and has 
the following properties:

(i) if $\sigma$ is closed, then $E_{\sigma} x=x$ if and only if $\sigma(x) \subset \sigma$;

(ii) $\sigma\left(E_{\sigma} x\right) \subset \bar{\sigma} \sigma(x), \sigma \in B, x \in X$.

To prove the sufficiency of the conditions it is, in view of 1.34 , sufficient to show that $\sigma\left(X_{e}\right) \subset \bar{e}, e \in B$, where $X_{e}=E_{e} X$. Let $\xi$ be a complex number not in $\bar{e} \sigma(T)$, and with each $\lambda \in \bar{e} \sigma(T)$ associate an $s_{3}$ set $\sigma_{\lambda}$ whose diameter is less than $1 / 2$ the distance from $\xi$ to $\bar{e} \sigma(T)$ and such that $\lambda$ is in the interior of $\sigma_{\lambda}$. A finite number $\sigma_{1}, \cdots, \sigma_{n}$ of these sets $\sigma_{\lambda}$ covers $\bar{e} \sigma(T)$, and since $s_{3}$ is a Boolean algebra the set $\sigma=\bigcup_{i=1}^{n} \sigma_{i} \in s_{3}$. Since $s_{3} \subset s_{1}$, we see from 1.21 that $\sigma\left(X_{\sigma}\right) \subset \bar{\sigma}$. But since

$$
E_{e}=E_{e} E_{\sigma(T)}=E_{e \sigma(T)} \subset E_{\bar{e} \sigma(T)} \subset E_{\sigma},
$$

we have $X_{e} \subset X_{\sigma}$. Since $\xi \not \subset \bar{\sigma}$ and $\sigma\left(X_{\sigma}\right) \subset \bar{\sigma}$, the operator $\xi-T$ is one-toone on $X_{\sigma}$ to all of $X_{\sigma}$, and hence likewise on the invariant subspace $X_{e}$. Thus

$$
\xi \in \rho\left(X_{e}\right),(\bar{e} \sigma(T))^{\circ} \subset \rho\left(X_{e}\right), \bar{e} \supset \bar{e} \sigma(T) \supset \sigma\left(X_{e}\right) .
$$

It will now be shown that if $T$ has a resolution of the identity $E_{e}$, then it is unique. Let $A_{e}$ also be a resolution of the identity for $T$. Let $\sigma, \sigma_{1}$ be disjoint closed sets of complex numbers. Since $\sigma\left(E_{\sigma_{1}} X\right) \subset \sigma_{1}$, the function $T(\xi) E_{\sigma_{1}} x$ analytic on $\rho(T)$ has an analytic extension to $\sigma_{1}^{\prime}$. Hence also the function

$$
T(\xi) A_{\sigma} E_{\sigma_{1}} x=A_{\sigma} T(\xi) E_{\sigma_{1}} x
$$

analytic on $\rho(T)$ has an analytic extension to $\sigma_{1}^{\prime}$. Since $\sigma\left(A_{\sigma} X\right) \subset \sigma$, the function $T(\xi) A_{\sigma} E_{\sigma_{1}} x$ has an analytic extension to $\sigma^{\prime}$. Thus

$$
\rho\left(A_{\sigma} E_{\sigma_{1}} x\right) \supset \sigma_{1}^{\prime} \cup \sigma^{\prime}=\left(\sigma_{1} \sigma\right)^{\prime}=\text { the whole plane; }
$$

that is, $\sigma\left(A_{\sigma} E_{\sigma_{1}} x\right)$ is void. By 1.12 we have $A_{\sigma} E_{\sigma_{1}}=0$. Likewise $E_{\sigma_{1}} A_{\sigma}=0$. Now there are closed sets $\sigma_{n} \subset \sigma_{n+1} \longrightarrow \sigma_{1}^{\prime}$, and hence $A_{\sigma_{n}} x \longrightarrow A_{\sigma_{1}^{\prime}} x=A_{\sigma_{1}^{\prime}} x$. Then

$$
0=A_{\sigma_{n}} E_{\sigma_{1}} x=E_{\sigma_{1}} A_{\sigma_{n}} x \longrightarrow E_{\sigma_{1}} A_{\sigma_{1}}^{\prime} x=A_{\sigma_{1}}^{\prime} E_{\sigma_{1}} x
$$

So

$$
0=E_{\sigma_{1}}\left(I-A_{\sigma_{1}}\right)=\left(I-A_{\sigma_{1}}\right) E_{\sigma_{1}}, E_{\sigma_{1}}=E_{\sigma_{1}} A_{\sigma_{1}}=A_{\sigma_{1}} E_{\sigma_{1}} \cdot
$$


Similarly,

$$
A_{\sigma_{1}}=E_{\sigma_{1}} A_{\sigma_{1}}=E_{\sigma_{1}} .
$$

Since $A_{\sigma} x$ and $E_{\sigma} x$ are both countably additive on $B$ and coincide for closed sets $\sigma$, they must coincide for all $\sigma \in B$. We shall now show that if $T$ has a resolution of the identity $E_{e}$, and $\sigma$ is closed, then $E_{\sigma} x=x$ if and only if $\sigma(x) \subset \sigma$. Let $\sigma$ be closed and $E_{\sigma} x=x$. Since $\sigma\left(X_{\sigma}\right) \subset \sigma$, the function

$$
T(\xi) E_{\sigma} x=T(\xi) x
$$

analytic on $\rho(T)$ has an analytic extension to $\sigma^{\prime}$. Thus $\rho(x) \supset \sigma^{\prime}, \sigma(x) \subset \sigma$. Conversely, let $\sigma(x) \subset \sigma$, where $\sigma$ is closed. Let $\sigma_{n}$ be closed, $\sigma_{n} \subset \sigma_{n+1} \longrightarrow$ $\sigma^{\prime}$, so that

$$
x=E_{\sigma} x+E_{\sigma}^{\prime} x=E_{\sigma} x+E_{\sigma} \cdot x=E_{\sigma} x+\lim _{n} E_{\sigma_{n}} x .
$$

Since $\sigma_{n} \sigma(T)$ and $\sigma$ are disjoint, closed, and $\sigma_{n} \sigma(T)$ is bounded, there is an admissible contour $C_{n}$ surrounding $\sigma_{n} \sigma(T)$ and excluding $\sigma$. Also, since

$$
\sigma\left(X_{\sigma_{n}}\right) \subset \sigma_{n} \sigma(T),
$$

we have

$$
E_{\sigma_{n}} x=\frac{1}{2 \pi i} \int_{C_{n}}\left(E_{\sigma_{n}} x\right)(\xi) d \xi=\frac{1}{2 \pi i} \int_{C_{n}} E_{\sigma_{n}} x(\xi) d \xi
$$

However, since $\sigma(x) \subset \sigma$, the function $x(\xi)$ is analytic on and within $C_{n}$. Thus

$$
E_{\sigma_{n}} x=0 \text { and } x=E_{\sigma} x .
$$

In this proof we have used the equality

$$
\left(E_{\sigma_{n}} x\right)(\xi)=E_{\sigma_{n}} x(\xi),
$$

which is clear from 1.2 since both functions are analytic extensions of

$$
T(\xi) E_{\sigma_{n}} x=E_{\sigma_{n}} T(\xi) x
$$

on $\rho(T)$. We shall next show that $\sigma\left(E_{\sigma} x\right) \subset \bar{\sigma} \sigma(x)$ for every $\sigma \in B$ and $x \in X$. Since $\sigma\left(X_{\sigma}\right) \subset \bar{\sigma}$, it is clear that $\sigma\left(E_{\sigma} x\right) \subset \bar{\sigma}$. Since 


$$
\left(E_{\sigma} x\right)(\xi)=E_{\sigma} x(\xi) \text { for } \xi \in \rho(T),
$$

we have $\rho\left(E_{\sigma} x\right) \supset \rho(x), \sigma\left(E_{\sigma} x\right) \subset \sigma(x)$. Thus $\sigma\left(E_{\sigma} x\right) \subset \bar{\sigma} \sigma(x)$. The necessity of 1.7 follows immediately from (i). Next we shall show the necessity of 1.14. As pointed out in 1.36 (ii) it follows from the principle of uniform boundedness that

$$
\sup _{e \in B}\left|E_{e}\right| \equiv K<\infty
$$

Let $\sigma=\sigma(x), \sigma_{1}=\sigma(y)$ be disjoint. Then, by (i), $E_{\sigma} x=x$; and, by (ii) and 1.12, $E_{\sigma} y=0$. Thus

$$
|x|=\left|E_{\sigma}(x+y)\right| \leq K|x+y| \text {. }
$$

Finally we prove the necessity of 1.39. Let $\sigma$ be a circle and its interior. Let $\sigma_{n}$ be closed and $\sigma_{n} \subset \sigma_{n+1} \rightarrow \sigma^{\prime}$. Then $E_{\sigma} x+E_{\sigma_{n}} x \rightarrow x$. Since

$$
\sigma\left(E_{\sigma} x\right) \subset \sigma \sigma(x) \text { and } \sigma\left(E_{\sigma_{n}} x\right) \subset \sigma_{n} \sigma(x) \subset \sigma^{\prime} \sigma(x) \text {, }
$$

we see that $\sigma$ is an $s_{3}$ set.

\section{Operators whose spectra lie in a rectifiable Jordan curve}

In order to apply the final lemma of $\S 1$ we find it necessary to restrict further the nature of the spectrum $\sigma(T)$. Later we shall be interested in specific cases where the spectrum lies either in a straight line segment or in a circle, and these two cases may be treated simultaneously by restricting the spectrum in the manner described in the next paragraph. When this is done and a rate of growth is imposed upon the resolvent (Assumption 2.1), it is possible to give conditions, of a nature much more applicable than those of the preceding lemma, which will ensure the existence of a resolution of the identity. This may be accomplished in a variety of ways, and some of the sets of conditions given are necessary as well as sufficient.

Throughout $\S_{2}$ it is assumed that the spectrum $\sigma(T)$ is contained in a closed rectifiable Jordan curve $\Gamma_{0}$. In order to be able to manipulate in a fairly simple fashion the analytical operations involved, we suppose further that $\Gamma_{0}$ is embedded in a one parameter family $\Gamma_{\delta}\left(-\delta_{0} \leq \delta \leq \delta_{0}, 0<\delta_{0} \leq 1 / 2\right)$ of closed rectifiable Jordan curves, with $\Gamma_{\delta_{1}}$ interior to $\Gamma_{\delta_{2}}$ for $-\delta_{0} \leq \delta_{1}<\delta_{2} \leq$ $\delta_{0}$. The curve $\Gamma_{\delta}$ is defined by a function

$$
\xi=\xi(\lambda, \delta),-1 \leq \lambda \leq 1 \text {, with } \xi(-1, \delta)=\xi(1, \delta) .
$$


We suppose that the parameter $\delta$ has been chosen in such a way that $|\delta|$ is the distance measured along the arc $\xi(\lambda, \delta)$ from the point $\xi(\lambda, 0)$ to the point $\xi(\lambda, \delta)$, and that the $\operatorname{arcs} \xi(\lambda, \delta),-\delta_{0} \leq \delta \leq \delta_{0}$, for different values of $\lambda$, do not intersect. Finally we suppose that for each $\delta \in\left[-\delta_{0}, \delta_{0}\right]$ the point $\xi(\lambda, \delta)$ traces, as $\lambda$ increases from -1 to +1 , the curve $\Gamma_{\delta}$ in a counterclockwise direction.

2.1. AsSUMPTION. The spectrum $\sigma(T)$ of $T$ is contained in the rectifiable Jordan curve $\Gamma_{0}$ described above, and the rate of growth of the resolvent $T(\xi)$ for $\xi=\xi(\lambda, \delta)$ near the spectrum is restricted by the condition

$$
\limsup _{\delta \rightarrow 0}\left|\delta^{\nu(\lambda)} T(\xi)\right|<\infty,-1 \leq \lambda \leq 1
$$

where $\nu(\lambda)$ is a nonnegative function defined for $-1 \leq \lambda \leq 1$.

Since the function $\nu(\lambda)$ may be increased without destroying the above property, and since $\delta_{0} \leq 1 / 2$, every operator $T$ satisfying 2.1 has an index function $\nu(\lambda)$ according to the following definition.

2.2. DEFINITION. Any nonnegative integer-valued function $\nu(\lambda)$ satisfying the condition

$$
\left|\delta^{\nu(\lambda)} T(\xi)\right| \leq 1,0<|\delta|<\delta_{0}, \lambda \in[-1,1]
$$

will be called an index function for $T$.

It might be pointed out that if $\nu(\lambda)$ is defined only on the set $\Lambda \subset[-1,1]$ consisting of all those $\lambda$ for which $\xi(\lambda, 0) \in \sigma(T)$, and the above inequality is valid for $\lambda \in \Lambda$, then $T$ has an index function. It is not assumed that $\nu(\lambda)$ is bounded, and it is erroneous to conclude that $T$ has a bounded index function providing $\nu(\lambda)$ is bounded on $\Lambda$. Elementary operators exist for which every index function is unbounded and at the same time every index function is bounded on $\Lambda$.

2.3. LеммA. (Assumption 2.1.) There is an index function $\nu(\lambda)$ for $T$ with the property that every interval of positive length contains an interval of positive length upon which $\nu(\lambda)$ is constant.

Let $\Delta$ be a closed subinterval of $[-1,1]$. Let $\Delta_{n}$ be the set of all $\lambda \in \Delta$ such that

$$
\left|\delta^{n} T(\xi)\right| \leq 1,0<|\delta| \leq \delta_{0} .
$$


Since for fixed $\delta \neq 0$ the point $\xi=\xi(\lambda, \delta)$, and thus $T(\xi)$, is continuous in $\lambda$, it follows that $\Delta_{n}$ is closed. By 2.1 we see that $\Delta=U \Delta_{n}$, and thus the desired conclusion follows from the category theorem of Baire.

2.4. LemmA. (Assumption 2.1.) Let $\nu(\lambda)$ be an index function for $T: 0<$ $\delta \leq \delta_{0} ; \lambda_{1}, \lambda_{2} \in[-1,1] ; \xi_{1}=\xi\left(\lambda_{1}, 0\right), \xi_{2}=\xi\left(\lambda_{2}, 0\right)$ distinct points of $\Gamma_{0}$; and $\nu_{1}=\nu\left(\lambda_{1}\right), \nu_{2}=\nu\left(\lambda_{2}\right)$. Let $C\left(\lambda_{1}, \lambda_{2}\right)$ be the rectifiable contour (oriented counterclockwise) composed of the following four arcs. There are two cases: If $\lambda_{1}<\lambda_{2}$ the arcs are

$$
\begin{aligned}
& \xi\left(\lambda_{1}, \mu\right),-\delta \leq \mu \leq \delta ; \quad \xi\left(\lambda_{2}, \mu\right),-\delta \leq \mu \leq \delta ; \\
& \xi(\lambda, \delta), \lambda_{1} \leq \lambda \leq \lambda_{2} ; \quad \text { and } \quad \xi(\lambda,-\delta), \lambda_{1} \leq \lambda \leq \lambda_{2} ;
\end{aligned}
$$

whereas if $\lambda_{2}<\lambda_{1}$ we use the arcs

$$
\begin{aligned}
& \xi\left(\lambda_{1}, \mu\right),-\delta \leq \mu \leq \delta ; \quad \xi\left(\lambda_{2}, \mu\right),-\delta \leq \mu \leq \delta ; \\
& \xi(\lambda, \delta), \lambda \notin\left(\lambda_{2}, \lambda_{1}\right) ; \text { and } \xi(\lambda, \delta), \lambda \notin\left(\lambda_{2}, \lambda_{1}\right) .
\end{aligned}
$$

Let $P(\xi)$ be a polynomial in $\xi$. Then

$$
I\left(\lambda, \lambda_{2}\right)=\frac{1}{2 \pi i} \int_{C\left(\lambda_{1}, \lambda_{2}\right)} P(\xi)\left(\xi-\xi_{1}\right)^{\nu_{1}}\left(\xi-\xi_{2}\right)^{\nu_{2}} T(\xi) d \xi
$$

exists as a Riemann integral, is independent of $\delta$, and has the properties

$$
\lim _{0<\left|\lambda_{2}-\lambda_{1}\right| \rightarrow 0} I\left(\lambda_{1}, \lambda_{2}\right)=0, \quad \sigma\left(I\left(\lambda_{1}, \lambda_{2}\right) x\right) \subset\left[\xi_{1}, \xi_{2}\right],
$$

where $\left[\xi_{1}, \xi_{2}\right]$ is the closed subarc of $\Gamma_{\theta}$ consisting of all points of $\Gamma_{0}$ which are inside or on the contour $C\left(\lambda_{1}, \lambda_{2}\right)$.

The integrand is defined and continuous at every point of the contour $C\left(\lambda_{1}\right.$, $\lambda_{2}$ ) except at the points $\xi_{1}$ and $\xi_{2}$. Since $\nu(\lambda)$ is an index function for $T$, the integrand is bounded on the curve $C\left(\lambda_{1}, \lambda_{2}\right)$. Hence $I\left(\lambda_{1}, \lambda_{2}\right)$ exists. It is clearly independent of $\delta$ since the integrand has its only singularities on the curve $\Gamma_{0}$. Now let $\lambda_{1}<\lambda_{2}$ so that $C\left(\lambda_{1}, \lambda_{2}\right)$ consists of the $\operatorname{arcs} A B, B C, C D$, $D A$, where $A, B, C, D$ are given by the complex numbers

$$
\xi\left(\lambda_{2},-\delta\right), \xi\left(\lambda_{1},-\delta\right), \xi\left(\lambda_{1}, \delta\right), \xi\left(\lambda_{2}, \delta\right) .
$$




$$
K=\text { l.u.b. }|P(\xi)|, \xi \in \Gamma_{\delta_{0}} ; K_{\delta}=\text { l.u.b. }|T(\xi)|, \quad \xi \in \Gamma_{\delta} \text {. }
$$

Let $0<\epsilon<\delta_{0}, \delta=\epsilon / 2$, and let $\gamma_{\epsilon}$ be such that for $0<\lambda_{2}-\lambda_{1}<\gamma_{\epsilon}$ we have the lengths of the $\operatorname{arcs} A B, C D$, and $\xi_{1} \xi_{2}$ (on $\Gamma_{0}$ ) all less than $\delta$ and also less than $\epsilon / K(\delta)$. Then the integrand $f(\xi)$ defining $I\left(\lambda_{1}, \lambda_{2}\right)$ for $\xi=\xi(\lambda, \mu)$ satisfies

$$
|f(\xi)| \leq K\left|\xi-\xi_{2}\right|^{\nu_{2}}|\mu|^{\nu_{1}}|T(\xi)| \leq K\left|\xi-\xi_{2}\right|^{\nu_{2}}, \xi \in B C .
$$

Since, for $\xi \in B C$, we have

$$
\left|\xi-\xi_{2}\right| \leq\left|\xi_{2}-\xi_{1}\right|+\left|\xi_{1}-\xi\right| \leq \delta+\mu \leq 2 \delta<1,
$$

it follows that for $\xi \in B C$, and likewise for $\xi \in D A$, we have the bound

$$
|f(\xi)| \leq K
$$

For $\xi$ on $A B$ or $C D$, we have

$$
|f(\xi)| \leq K K_{\delta}\left|\xi-\xi_{2}\right|^{\nu_{2}}\left|\xi-\xi_{1}\right|^{\nu_{1}} \leq K K_{\delta} .
$$

Thus if $0<\lambda_{2}-\lambda_{1}<\gamma_{\epsilon}$, then

$$
\left|I\left(\lambda_{1}, \lambda_{2}\right)\right| \leq \frac{1}{2 \pi}\left[4 \delta K+2 K K_{\delta} \epsilon / K_{\delta}\right]=2 K \epsilon / \pi .
$$

Now let $x \in X, \eta \in \rho(T)$, and $\eta$ outside of $C\left(\lambda_{1}, \lambda_{2}\right)$. Then

$T(\eta) I\left(\lambda_{1}, \lambda_{2}\right) x$

$$
\begin{aligned}
& =\frac{1}{2 \pi i} \int_{C\left(\lambda_{1}, \lambda_{2}\right)} P(\xi)\left(\xi-\xi_{1}\right)^{\nu_{1}}\left(\xi-\xi_{2}\right)^{\nu_{2}}(\eta-\xi)^{-1} T(\xi) x d \xi \\
& \quad+\frac{1}{2 \pi i} T(\eta) x \int_{C\left(\lambda_{1}, \lambda_{2}\right)} P(\xi)\left(\xi-\xi_{1}\right)^{\nu_{1}}\left(\xi-\xi_{2}\right)^{\nu_{2}}(\eta-\xi)^{-1} d \xi \\
& =\frac{1}{2 \pi i} \int_{C\left(\lambda_{1}, \lambda_{2}\right)} P(\xi)\left(\xi-\xi_{1}\right)^{\nu_{1}}\left(\xi-\xi_{2}\right)^{\nu_{2}}(\eta-\xi)^{-1} T(\xi) x d \xi,
\end{aligned}
$$

and the last integral gives an analytic extension of $T(\eta) I\left(\lambda_{1}, \lambda_{2}\right) x$ for all $\eta$ outside $C\left(\lambda_{1}, \lambda_{2}\right)$. 
2.5. Lemma. (Assumption 2.1.) The operator $T$ satisfies the conditions 1.1 and 1.7 .

Condition 1.1 clearly is satisfied since a rectifiable Jordan curve is nondense in the plane. To prove 1.7, let $\sigma$ be a closed subset of the spectrum, $x_{n} \in[\sigma], x_{n} \rightarrow x_{0}$. We make an indirect proof by supposing that there is a point $\xi \in \sigma(x) \sigma^{\prime}$. According to the Heine-Borel theorem there are closed disjoint subarcs $\Delta_{1}, \cdots, \Delta_{p}$ of $\Gamma_{0}$ with $\sigma \subset \Delta=\Delta_{1} \cup \cdots \cup \Delta_{p}$ and $\xi \in \Delta^{\prime}$. Let

$$
-1 \leq \lambda_{1}<\mu_{1}<\lambda_{2}<\mu_{2}<\cdots<\lambda_{p}<\mu_{p}<1
$$

be such that the arc $\Delta_{j},(j=1, \cdots, p)$ is defined by $\xi(\lambda, 0), \lambda_{j} \leq \lambda \leq \mu_{j}$. Let $C_{j}=C\left(\lambda_{j}, \mu_{j}\right)$ as defined in 2.4. Since $x_{n}(\xi)$ has its singularities in the set $\Delta$, we see that

$$
\int_{C} \prod_{j=1}^{p}\left(\xi-\xi_{j}\right)^{\nu\left(\lambda_{j}\right)}\left(\xi-\zeta_{j}\right)^{\nu\left(\mu_{j}\right)} x_{n}(\xi) d \xi=0,
$$

where

$$
\xi_{j}=\xi\left(\lambda_{j}, 0\right), \quad \zeta_{j}=\xi\left(\mu_{j}, 0\right)
$$

and $C$ is any contour of the form $C=C\left(\lambda_{1}, \lambda_{2}\right)$ providing $\left[\lambda_{1}, \lambda_{2}\right]$ is disjoint with $\Delta$. Since, by 2.4 ,

$$
I\left(\lambda_{j}-\frac{1}{n}, \lambda_{j}\right) \rightarrow 0, I\left(\mu_{j}, \mu_{j}+\frac{1}{n}\right) \rightarrow 0,
$$

we have,

$$
\begin{aligned}
& \prod_{j=1}^{p}\left(T-\xi_{j}\right)^{\nu\left(\lambda_{j}\right)}\left(T-\zeta_{j}\right)^{\nu\left(\mu_{j}\right)} x_{n} \\
& \quad=\frac{1}{2 \pi i} \sum_{j=1}^{p} \int_{C_{j}} \prod_{k=1}^{p}\left(\xi-\xi_{k}\right)^{\nu\left(\lambda_{k}\right)}\left(\xi-\zeta_{k}\right)^{\nu\left(\mu_{k}\right)} x_{n}(\xi) d \xi .
\end{aligned}
$$

Since the convergence, as $n \longrightarrow \infty$, of the integrands on the right side of the above equality is bounded, we may in this equation replace $x_{n}$ by $x$ and conclude from 2.4 and 1.6 that 


$$
\sigma\left(\prod_{j=1}^{p}\left(T-\xi_{j}\right)^{\nu\left(\lambda_{j}\right)}\left(T-\zeta_{j}\right)^{\nu\left(\mu_{j}\right)} x\right) \subset \Delta .
$$

The desired contradiction will be obtained as soon as we show that the above inclusion implies that $\sigma(x) \subset \Delta$. But this implication follows immediately by induction from the statement

$$
\sigma(z) \subset \sigma((\xi-T) z) \cup(\xi),
$$

which is verified as follows. Since

$$
T(\mu) z=(\xi-\mu)^{-1}\{T(\mu)(\xi-T) z-z\}, \mu \neq \xi,
$$

any point $\mu$ other than $\mu=\xi$ to which $T(\mu)(\xi-T) z$ has an analytic extension must be in $\rho(z)$; that is,

$$
\rho((\xi-T) z) \subset \rho(z) \cup(\xi) .
$$

Thus

$$
\sigma((\xi-T) z) \supset \sigma(z) \cap(\xi)^{\prime} \cdot
$$

2.6. Lemma. (Assumption 2.1.) Let $\nu(\lambda)$ be an index function for T. For every complex number $\xi$ and every nonnegative integer $n$, define

$$
\mathbb{M}_{\xi}^{n}=\varepsilon_{x}\left[(T-\xi)^{n} x=0\right], \Re_{\xi}^{n}=(T-\xi)^{n} X
$$

Then for $\xi=\xi(\lambda, 0)$ we have

$$
\mathbb{M}_{\xi}^{\nu(\lambda)}=\Re_{\xi}^{n}, \bar{\Re}_{\xi}^{\nu(\lambda)}=\bar{\Re}_{\xi}^{n}, \quad n \geq \nu(\lambda) .
$$

Let $\xi_{1}=\xi(\lambda, \delta)$; then

$$
(\xi-T) T\left(\xi_{1}\right)=\left(\xi-\xi_{1}\right) T\left(\xi_{1}\right)+I .
$$

Now assume, for the purpose of induction, that (the preceeding identity is the case when $j=1$ )

$$
\begin{array}{r}
(\xi-T)^{j} T\left(\xi_{1}\right)=\left(\xi-\xi_{1}\right)^{j} T\left(\xi_{1}\right)+\left(\xi-\xi_{1}\right)^{j-1}+\left(\xi-\xi_{1}\right)^{j-2}(\xi-T) \\
+\cdots+\left(\xi-\xi_{1}\right)(\xi-T)^{j-2}+(\xi-T)^{j-1} .
\end{array}
$$


Multiplying this by $\xi-T$, we have

$$
\begin{array}{r}
(\xi-T)^{j+1} T\left(\xi_{1}\right)=\left(\xi-\xi_{1}\right)^{j}\left[\left(\xi-\xi_{1}\right) T\left(\xi_{1}\right)+I\right]+\left(\xi-\xi_{1}\right)^{j-1}(\xi-T) \\
+\cdots+\left(\xi-\xi_{1}\right)(\xi-T)^{j-1}+(\xi-T)^{j},
\end{array}
$$

and hence

(*) $\quad(\xi-T)^{j+1} T\left(\xi_{1}\right)=\left(\xi-\xi_{1}\right)^{j+1} T\left(\xi_{1}\right)+\left(\xi-\xi_{1}\right)^{j}$

$$
+\left(\xi-\xi_{1}\right)^{j-1}(\xi-T)+\cdots+\left(\xi-\xi_{1}\right)(\xi-T)^{j-1}+(\xi-T)^{j} .
$$

Now in $\left(^{*}\right)$ put $j=\nu(\lambda)$, and operate on a vector $x \in \mathbb{R}_{\xi}^{\nu(\lambda)+1}$. We get

$$
\begin{aligned}
0=\left(\xi-\xi_{1}\right)^{\nu(\lambda)+1} T\left(\xi_{1}\right) x & +\left(\xi-\xi_{1}\right)^{\nu(\lambda)} x \\
& +\cdots+\left(\xi-\xi_{1}\right)(\xi-T)^{\nu(\lambda)-1} x+(\xi-T)^{\nu(\lambda)} x .
\end{aligned}
$$

If we let $\delta \rightarrow 0$, then $\xi_{1} \rightarrow \xi$; and since $\delta$ measures the arc $\xi \xi_{1}$, we have

$$
\left|\left(\xi-\xi_{1}\right)^{\nu(\lambda)+1} T\left(\xi_{1}\right) x\right|=\left|\delta^{\nu(\lambda)+1} T\left(\xi_{1}\right)\left(\frac{\xi-\xi_{1}}{\delta}\right)^{\nu(\lambda)+1}\right| \leq \delta \rightarrow 0 .
$$

This shows that $x \in \Re_{\xi}^{\nu(\lambda)}$. Thus

$$
\mathfrak{M}_{\xi}^{\nu(\lambda)+1} \subset \mathbb{M}_{\xi}^{\nu(\lambda)}
$$

and hence

$$
\mathbb{M}_{\xi}^{\nu(\lambda)}=\mathbb{M}_{\xi}^{n}, \quad n \geq \nu(\lambda)
$$

Using $(*)$ again except now with $x$ arbitrary, we may write

$$
(\xi-T)^{\nu(\lambda)} x=(\xi-T)^{\nu(\lambda)+1} T\left(\xi_{1}\right) x+O(\delta),
$$

where $O(\delta)$ is a vector which approaches zero with $\delta$. Thus

$$
\Re_{\xi}^{\nu(\lambda)} \subset \bar{\Re} \bar{\xi}_{\xi}^{\nu(\lambda)+1} \subset \bar{\Re} \bar{\Re}_{\xi}^{\nu(\lambda)}
$$

and so 


$$
\bar{\Re}_{\xi}^{\nu(\lambda)}=\bar{\Re}_{\xi}^{n}, n \geq \nu(\lambda)
$$

2.7. Definition. (Assumption 2.1.) Let $\nu(\lambda)$ be an index function for $T$. As shown in 2.6, the manifolds $\Re_{\xi}^{\nu(\lambda)}$ and $\bar{\Omega}_{\xi}^{\nu(\lambda)}$ are independent of the index function $\nu(\lambda)$. They will henceforth be designated by the symbols $\mathfrak{M}_{\xi}$, $\Re_{\xi}$, respectively.

Of the three principal conditions of Lemma 1.38, namely $1.7,1.14$, and 1.39, the condition 1.7 has already been shown (by 2.5 ) to be a consequence of 2.1. Neither one of the other two conditions is a consequence of 2.1 alone, and we concentrate our attention now on restating 1.39 in a more applicable form. The following assumption 2.8, which may be used to replace 1.39 , turns out to be necessary as well as sufficient. It has the disadvantage of not always being easily applicable. It should be noted, however, that in the case of operators with only continuous spectra it is trivially satisfied. Or more generally, if every subarc of $\Gamma_{0}$ contains points either in the resolvent set or in the continuous spectrum then 2.8 is automatically satisfied.

2.8. Assumption. (See Assumption 2.1.) For every $\xi$ in a dense set on $\Gamma_{0}$,

$$
\mathbb{M}_{\xi}+\mathfrak{N}_{\xi} \text { is dense in } X \text {. }
$$

2.9. Lemma. (Assumptions 2.1, 1.14.) The set of points $\xi$ on the curve $\Gamma_{0}$ for which $\mathbb{M}_{\xi} \Re_{\xi} \neq 0$ is nondense on $\Gamma_{0}$. Moreover, $\mathbb{M}_{\xi} \Re_{\xi}=0$ for every $\xi$, interior to a subarc of $\Gamma_{0}$ upon which some index function is constant. For such $\xi$, the set $\Re_{\xi} \oplus \Re_{\xi}$ is closed. Thus if 2.8 is satisfied then

$$
\mathfrak{M}_{\xi} \oplus \mathfrak{N}_{\xi}=X
$$

for every $\xi$ in a set dense on $\Gamma_{0}$.

In view of 2.3 , the first statement is a consequence of the second. Accordingly, let $\nu(\lambda)$ be an index function which is constant on the interval $\left[\lambda_{1}, \lambda_{2}\right]$, and let $\xi=\xi(\lambda, 0)$ where $\lambda_{1}<\lambda<\lambda_{2}$. Let

$$
\nu=\nu(\lambda), \xi_{n}=\xi\left(\lambda_{n}, 0\right), \zeta_{n}=\xi\left(\mu_{n}, 0\right),
$$

where

$$
\lambda_{1}<\lambda_{n}<\lambda<\mu_{n}<\lambda_{2} \text { and } \lambda_{n} \rightarrow \lambda, \quad \mu_{n} \rightarrow \lambda \text {. }
$$


Take $P(\xi) \equiv 1$ in 2.4 , so that

$$
I\left(\lambda_{n}, \mu_{n}\right)+I\left(\mu_{n}, \lambda_{n}\right)=\left(T-\xi_{n}\right)^{\nu}\left(T-\zeta_{n}\right)^{\nu} \rightarrow(T-\xi)^{2 \nu} .
$$

Thus, it is seen from 2.4 that the vector $(T-\xi)^{2 \nu} x$ is the limit of vectors $x_{n}=I\left(\mu_{n}, \lambda_{n}\right) x$ and $\xi \in \rho\left(x_{n}\right)$. Thus 2.6 and 2.7 show that every vector $x \in \Re_{\xi}$ is the limit of a sequence $\left\{x_{n}\right\}$ with $\xi \in \rho\left(x_{n}\right)$. Now if $x \in \Re_{\xi} \Re_{\xi}$ we have $(T-\xi)^{\nu} x=0$, and hence $\sigma(x) \subset(\xi)$. If $\xi \in \rho\left(x_{n}\right)$ and $x_{n} \longrightarrow x$, then $\xi \in \rho\left(-x_{n}\right)$; and, by 1.14,

$$
|x| \leq K\left|x-x_{n}\right| \rightarrow 0, x=0 \text {, and } \mathbb{M}_{\xi} \Re_{\xi}=0 .
$$

Finally we show that $\mathbb{M}_{\xi} \oplus \Re_{\xi}$ is closed. Let

$$
x_{n}+y_{n} \rightarrow z \text {, where } x_{n} \in \Re_{\xi}, y_{n} \in \Re_{\xi} .
$$

Since $y_{n}-y_{m} \in \Re_{\xi}$, there are vectors $u_{n m}$ with

$$
\left|y_{n}-y_{m}-u_{n m}\right|<(n m)^{-1}, \xi \in \rho\left(u_{n m}\right) \text {. }
$$

By 1.14, then,

$$
\left|x_{n}-x_{m}\right| \leq K\left|x_{n}-x_{m}-u_{n m}\right| \leq K\left\{\left|x_{n}-x_{m}+y_{n}-y_{m}\right|+(n m)^{-1}\right\} \rightarrow 0 .
$$

2.10. LemMA. (Assumptions $2.1,1.14,2.8$.$) For every pair \xi, \xi_{1}$ of distinct points in a set dense on $\Gamma_{0}$ we have

$$
M_{\xi} \oplus \mathbb{M}_{\xi_{1}} \oplus \mathfrak{S}_{\xi} \Re_{\xi_{1}}=X
$$

and $\Re_{\xi} \Re_{\xi_{1}}$ is the closure of the manifold $(T-\xi)^{\nu}\left(T-\xi_{1}\right)^{\nu_{1}} X$, where $\nu=\nu(\lambda), \nu_{1}=\nu\left(\lambda_{1}\right), \xi=\xi(\lambda, 0), \xi_{1}=\xi\left(\lambda_{1}, 0\right)$, and $\nu(\lambda)$ is an index function for $T$.

For every $\xi$ in a set $\Gamma$ dense on $\Gamma_{0}$ we have, by 2.9 , projections $A_{\xi}$ and $A_{\xi}^{\prime}$ with

$$
A_{\xi}+A_{\xi}^{\prime}=I, A_{\xi} X=\mathbb{M}_{\xi}, A_{\xi}^{\prime} X=\Re_{\xi} .
$$

Since for $\mu \in \rho(T)$, we have $T(\mu) \Re_{\xi} \subset \Re_{\xi}$ and $T(\mu) \Re_{\xi} \subset \Re_{\xi}$, it follows that $T(\mu) A_{\xi} x=A_{\xi} T(\mu) x$, and thus $\rho(x) \subset \stackrel{\xi}{\rho}\left(A_{\xi} x\right)$. Since 


$$
\left(T-\xi_{1}\right)^{\nu_{1}} A_{\xi_{1}}=0,
$$

we have $\sigma\left(A_{\xi_{1}} x\right) \subset\left(\xi_{1}\right)$, and hence $\xi \in \rho\left(A_{\xi_{1}} x\right) \subset \rho\left(A_{\xi} A_{\xi_{1}} x\right)$. Since $\sigma\left(A_{\xi} y\right) \subset(\xi)$, we have $y \in X$; then $A_{\xi} A_{\xi_{1}}=0$ by 1.12 and 2.5. Similarly $A_{\xi_{1}} A_{\xi}=0$. Thus

$$
I=\left(A_{\xi}+A_{\xi}^{\prime}\right)\left(A_{\xi_{1}}+A_{\xi_{1}}^{\prime}\right)=A_{\xi}+A_{\xi}^{\prime}+A_{\xi}^{\prime} A_{\xi_{1}}^{\prime},
$$

and this proves the first statement of the lemma. Now let $x \in \Re_{\xi}^{\nu} \Re_{\xi}^{\nu_{1}}$, so that

$$
x=(T-\xi)^{\nu} y, y=A_{\xi_{1}} y+A_{\xi_{1}}^{\prime} y, x=A_{\xi_{1}}^{\prime} x
$$

By 2.7 there are vectors $v_{n}$ with $\left(T-\xi_{1}\right)^{\nu_{1}} v_{n} \rightarrow A_{\xi_{1}}^{\prime} y$ and

$$
\begin{aligned}
& x=\lim _{n}(T-\xi)^{\nu}\left[A_{\xi_{1}} y+\left(T-\xi_{1}\right)^{\nu} v_{n}\right], \\
& x=A_{\xi_{1}}^{\prime} x=\lim _{n}(T-\xi)^{\nu}\left(T-\xi_{1}\right)^{\nu} v_{n} .
\end{aligned}
$$

Thus $\Re_{\xi}^{\nu} \Re_{\xi_{1}}^{\nu_{1}}$ as well as its closure $\Re_{\xi} \Re_{\xi_{1}}$ is contained in the closure

$$
(T-\xi)^{\nu}\left(T-\xi_{1}\right)^{\nu_{1}} X \text {. }
$$

Obviously $(T-\xi)^{\nu}\left(T-\xi_{1}\right)^{\nu_{1}} X \subset \Re_{\xi} \Re_{\xi_{1}}$, and so the proof is complete.

2.11. THEOREM. (Assumptions 1.14, 2.1, 2.8.) Every Borel set is measurable $T$; and if $X$ is weakly complete then $T$ has a resolution of the identity.

Let $-1 \leq \alpha<\beta<\gamma<\delta<1$, and choose $\lambda_{1}, \lambda_{2}, \mu_{1}, \mu_{2}$ so that

$$
\alpha<\lambda_{1}<\lambda_{2}<\beta, \quad \gamma<\mu_{1}<\mu_{2}<\delta,
$$

and such that there is an index function $\nu(\lambda)$ which is constant on the intervals $\left[\lambda_{1}, \lambda_{2}\right],\left[\mu_{1}, \mu_{2}\right]$. This is possible in view of 2.3. Since an index function may be increased without destroying the property of being an index function, we shall suppose that $\nu(\lambda)$ has the constant value $\nu$ on both of the intervals $\left[\lambda_{1}, \lambda_{2}\right],\left[\mu_{1}, \mu_{2}\right]$. Let 


$$
\xi_{1}=\xi\left(\lambda_{1}, 0\right), \xi_{2}=\xi\left(\lambda_{2}, 0\right), \zeta_{1}=\xi\left(\mu_{1}, 0\right), \zeta_{2}=\xi\left(\mu_{2}, 0\right),
$$

and

$$
f(\xi)=\left(\xi-\xi_{1}\right)^{\nu}\left(\xi-\xi_{2}\right)^{\nu}\left(\xi-\zeta_{1}\right)^{\nu}\left(\xi-\zeta_{2}\right)^{\nu}
$$

Then for appropriate choices of the polynomial $P(\xi)$ in 2.4 we have

$$
f(T)=I\left(\lambda_{1}, \lambda_{2}\right)+I\left(\mu_{1}, \mu_{2}\right)+I\left(\mu_{2}, \lambda_{1}\right)+I\left(\lambda_{2}, \mu_{1}\right) .
$$

By 2.10 there are points $\lambda$, $\mu$ with $\lambda_{1}<\lambda<\lambda_{2}, \mu_{1}<\mu<\mu_{2}$, and such that for $\xi=\xi(\lambda, 0), \zeta=\xi(\mu, 0)$ we have

$$
\mathfrak{M}_{\xi} \oplus \mathfrak{M}_{\zeta} \oplus \mathfrak{N}_{\xi} \mathfrak{N}_{\zeta}=X
$$

Now if we let $\lambda_{1} \rightarrow \lambda, \lambda_{2} \rightarrow \lambda, \mu_{1} \rightarrow \mu, \mu_{2} \rightarrow \mu$ then by 2.4 we have

$$
I\left(\lambda_{1}, \lambda_{2}\right) \longrightarrow 0, I\left(\mu_{1}, \mu_{2}\right) \rightarrow 0,
$$

and

$$
(T-\xi)^{2 \nu}(T-\zeta)^{2 \nu} x=\lim f(T) x=\lim \left\{I\left(\mu_{2}, \lambda_{1}\right) x+I\left(\lambda_{2}, \mu_{1}\right) x\right\}
$$

Also, by 2.4, we have $\sigma\left(I\left(\mu_{2}, \lambda_{1}\right) x\right) \subset\left[\zeta_{2}, \xi_{1}\right]$ and $\sigma\left(I\left(\lambda_{2}, \mu_{1}\right) x\right) \subset\left[\xi_{2}, \zeta_{1}\right]$, where, for two points $\xi^{\prime}=\xi\left(\lambda^{\prime}, 0\right), \xi^{\prime \prime}=\xi\left(\lambda^{\prime \prime}, 0\right)$ on $\Gamma_{0}$, the symbol $\left[\xi^{\prime}, \xi^{\prime \prime}\right]$ means the closed subarc of $\Gamma_{0}$ defined by $\xi(\lambda, 0), \lambda^{\prime} \leq \lambda \leq \lambda^{\prime \prime}$, if $\lambda^{\prime}<\lambda^{\prime \prime}$ and closed subarc $\xi(\lambda, 0), \lambda \notin\left(\lambda^{\prime \prime}, \lambda^{\prime}\right)$, if $\lambda^{\prime \prime}<\lambda^{\prime}$.

Since $2 \nu(\lambda)$ is also an index function, it is seen from 2.10 that $\Re_{\xi} \Re_{\zeta}$ is the closure of $(T-\xi)^{2 \nu}(T-\zeta)^{2 \nu} X$, and hence every vector in $\Re_{\xi}^{\zeta} \Re_{\zeta}^{\zeta}$ is the limit of a sequence of vectors of the form $x+y$ with $\sigma(x) \subset[\xi, \zeta], \sigma(y) \subset$ $[\xi, \zeta]^{\prime}$. Since $\sigma(x) \subset(\xi)$ if $x \in M_{\xi}$, we have, from 1.6 and $(*)$ above, the fact that every vector in $X$ is the limit of a sequence of vectors of the form $x+y$ with $\sigma(x) \subset[\xi, \zeta], \sigma(y) \subset[\xi, \zeta]^{\prime}$. This shows that $[\xi, \zeta]$ is an $s_{1}$ set for $T$ (see Definition 1.15). The above argument shows also that $[\zeta, \xi]$ is an $s_{1}$ set for $T$. We shall next show that $\sigma=[\xi, \zeta]$ is an $s_{2}$ set for $T$. If the intervals $(\alpha, \beta)$ and $(\alpha, \delta)$ that we started with above are replaced by the intervals $(\lambda-1 / n, \lambda)$ and $(\mu, \mu+1 / n)$, we see that there are points $\lambda_{n}, \mu_{n}$, with $\lambda-1 / n<\lambda_{n}<\lambda, \mu<\mu_{n}<\mu+1 / n$, such that $\sigma_{n} \equiv\left[\zeta_{n}, \xi_{n}\right]$, where

$$
\zeta=\xi\left(\mu_{n}, 0\right), \xi_{n}=\xi\left(\lambda_{n}, 0\right),
$$


is an $s_{1}$ set. Now let

$$
y=(T-\xi)^{2 \nu}(T-\zeta)^{2 \nu} x, y_{n}=(T-\xi)^{\nu}\left(T-\xi_{n}\right)^{\nu}(T-\zeta)^{\nu}\left(T-\zeta_{n}\right)^{\nu} x,
$$

so that $y_{n} \longrightarrow y$, and for appropriate choices of $P(\xi)$ in 2.4 we have

$$
y_{n}=I\left(\lambda_{n}, \lambda\right) x+I(\lambda, \mu) x+I\left(\mu, \mu_{n}\right) x+I\left(\mu_{n}, \lambda_{n}\right) x
$$

Thus by 2.4 we may write

$$
y=I(\lambda, \mu) x+I\left(\mu_{n}, \lambda_{n}\right) x+z_{n}, \text { where } z_{n} \rightarrow 0 .
$$

Now from $2.4,2.5$, and 1.16 we see that

$$
\begin{aligned}
& E_{\sigma} I(\lambda, \mu)=I(\lambda, \mu), E_{\sigma} I\left(\mu_{n}, \lambda_{n}\right)=0, \\
& E_{\sigma_{n}} I(\lambda, \mu)=0, E_{\sigma_{n}} l\left(\mu_{n}, \lambda_{n}\right)=I\left(\mu_{n}, \lambda_{n}\right) ;
\end{aligned}
$$

and since $\left|E_{\sigma_{n}}\right| \leq K$ we may write $y=E_{\sigma} y+E_{\sigma_{n}} y+v_{n}$ where $v_{n} \rightarrow 0$. Since $y$ is an arbitrary point in the manifold $(T-\xi)^{2 \nu}(T-\zeta)^{2 \nu} X$ whose closure is $\Re_{\xi} \Re_{\zeta}$, and since

$$
\left|E_{\sigma}+E_{\sigma_{n}}\right| \leq 2 K
$$

we have

$$
y=E_{\sigma} y+\lim _{n} E_{\sigma_{n}} y
$$

for every $y \in \Re_{\xi} \Re_{\zeta}$. For $x \in \Re_{\xi} \oplus M_{\zeta}$ we have $\sigma(x) \subset \sigma$; and so, by 1.16, we have $E_{\sigma} x=x, E_{\sigma_{n}} x=0$. Hence, it follows from $(*)$ that

$$
x=E_{\sigma} x+\lim _{n} E_{\sigma_{n}} x, x \in X .
$$

Now 2.5 and 1.22 show that $\sigma\left(E_{\sigma} x\right) \subset \sigma \sigma(x), \sigma\left(E_{\sigma_{n}} x\right) \subset \sigma_{n} \sigma(x) \subset \sigma^{\prime} \sigma(x)$, so that $\sigma$ is an $s_{2}$ set. The same argument shows that $\left[\zeta_{n}, \xi_{n}\right]=\sigma_{n}$ is an $s_{2}$ set and hence $(* *)$ shows that $\sigma$ is an $s_{3}$ set for $T$. Thus we have proved that if $-1 \leq \alpha<\beta<\gamma<\delta<1$ there are points $\lambda, \mu$ with $\alpha<\lambda<\beta, \gamma<\mu<\delta$, such that $\sigma=[\xi(\lambda, 0), \xi(\mu, 0)]$ is an $s_{3}$ set. This clearly implies the statement 1.39; hence every Borel set is measurable T. Theorem 2.11 then follows from 1.38. 


\section{The operational calculus}

3.1. Definition of $\int_{\sigma} f(\lambda) d E_{\lambda}$. In what follows we shall be concerned with an integral, $\int f(\lambda) d E_{\lambda}$, where $E_{e}$ is the resolution of the identity for an operator $T$. The functions $f$ to be integrated are either scalar- or operator-valued; they will always be continuous, so that the Riemann integral will suffice. Although the applications to be made are to operators satisfying the preceding restrictions, it seems desirable to word the definitions and elementary properties of $\int f(\lambda) d E_{\lambda}$ in terms of an arbitrary operator $T$ on an arbitrary space $X$ subject to the single restriction that $T$ has a resolution of the identity. Since $\sigma(T)$ is bounded, it may for any $\delta>0$ be partitioned into disjoint, nonvoid Borel sets $\Delta_{1}, \cdots, \Delta_{n}$ whose diameters are less than $\delta$. The norm $|\pi|$ of such a partitioning $\pi=\left(\Delta_{1}, \cdots, \Delta_{n}\right)$ is $|\pi|=\max _{i}$ diam $\Delta_{i}$. If for a scalar- or operator-valued function $f$ defined on $\sigma(T)$ we have the sums $\sum_{\pi} f\left(\lambda_{i}\right) E_{\Delta_{i}}$ converging, as $|\pi| \rightarrow 0$, to a limit independent of the choice of $\lambda_{i} \in \Delta_{i}$, the function $f$ is said to be integrable. Of course the convergence of $\sum_{\pi} f\left(\lambda_{i}\right) E_{\Lambda_{i}}$ as $|\pi| \rightarrow 0$ may be in the weak, strong, or uniform topology of operators; but for the functions we shall integrate, it is always in the uniform topology of operators, so we need not concern ourselves here with the other cases. The integral is defined by

$$
\int f(\lambda) d E_{\lambda} \equiv \lim _{|\pi| \rightarrow 0} \sum_{\pi} f\left(\lambda_{i}\right) E_{\Delta_{i}}
$$

and for any Borel set $\sigma$ in the plane we define $\int_{\sigma} f(\lambda) d E_{\lambda}$ to be $E_{\sigma} \int f(\lambda) d E_{\lambda}$.

3.2. LemmA. If for each $e$ in a Borel algebra $\beta$ there is a bounded linear operator $A_{e}$ in the space $X$ such that $x^{*} A_{e} x$ is countably additive on $\beta$ for each $x \in X, x^{*} \in X^{*}$, then there is a constant $v(A)$ such that

$$
\sum_{i}\left|x^{*} A_{e_{i}} x\right| \leq v(A)|x|\left|x^{*}\right|, e_{i} e_{j} \text { void for } i \neq j, e_{i} \in \beta
$$

Let $\pi=\left\{e_{i}\right\}$ be a finite or enumerable sequence of disjoint elements in $\beta$. For each $x \in X, x^{*} \in X^{*}$, define $U_{\pi}\left(x, x^{*}\right)$ as the point in the complex Banach space $\ell_{1}$ (the space of absolutely convergent sequences) given by the sequence $\left\{x^{*} A_{e_{i}} x\right\}$ (if the sequence $\left\{e_{i}\right\}$ is finite we extend it to an infinite sequence by taking $e_{n}$ to be the void set for all large $n$ ). For fixed $x, \pi$, the function $U_{\pi}\left(x, x^{*}\right)$ is additive, homogeneous, and closed; hence $U_{\pi}\left(x, x^{*}\right)$ is continuous in $x^{*}$. Similarly, $U_{\pi}\left(x, x^{*}\right)$ is continuous in $x$ for fixed $x^{*}, \pi$. Thus for each $\pi, U_{\pi}\left(x, x^{*}\right)$ is simultaneously continuous in $x, x^{*}$. Since the numerical function 
$x^{*} A_{e} x$ is countably additive on $\beta$, we have

$$
\sup _{\pi}\left|U_{\pi}\left(x, x^{*}\right)\right|<\infty, x \in X, x^{*} \in X^{*} .
$$

Let $Z_{n}$ be the set of points $\left(x, x^{*}\right)$ in the Cartesian product space $Z=X \times X^{*}$, where $\left|U_{\pi}\left(x, x^{*}\right)\right| \leq n$ for every $\pi$. Since $U_{\pi}\left(x, x^{*}\right)$ is continuous in $\left(x, x^{*}\right)$, $Z_{n}$ is closed. From $(*)$ we have $Z=\cup Z_{n}$, and so the Baire catagory theorem gives an integer $n_{0}$, a point $\left(x_{0}, x_{0}^{*}\right) \in Z$, and an $r_{0}>0$, such that

$$
\left|U_{\pi}\left(x, x^{*}\right)\right| \leq n_{0}, \pi,\left|x-x_{0}\right|<r_{0},\left|x^{*}-x_{0}^{*}\right|<r_{0} .
$$

Now if $y \in X, y^{*} \in X^{*}$, and $|y|,\left|y^{*}\right|<r_{0}$, we have

$$
\begin{aligned}
U_{\pi}\left(y, y^{*}\right) & =U_{\pi}\left(x_{0}-y, x_{0}^{*}-y^{*}\right)=U_{\pi}\left(x_{0}-y, x_{0}^{*}\right) \\
& =U_{\pi}\left(x_{0}, x_{0}^{*}-y^{*}\right)+U_{\pi}\left(x_{0}, \mathrm{x}_{0}^{*}\right),
\end{aligned}
$$

and $\mid U_{\pi}\left(y, y^{\prime \prime}\right) \leq 4 n_{0}$. Thus if $v(A)=5 n_{0} / r_{0}^{2}$, we have

$$
\left|U_{\pi}\left(y, y^{*}\right)\right| \leq v(A)|y|\left|y^{*}\right|
$$

3.3. LеммA. Every continuous scalar function $f$ on $\sigma(T)$ is integrable, and

$$
\begin{aligned}
& \left|\int f(\lambda) d E_{\lambda}\right| \leq \max _{\lambda \in \sigma(T)}|f(\lambda)| v(E), \\
& \left|\int_{\sigma} f(\lambda) d E_{\lambda}\right| \leq \sup _{\lambda \in \sigma}|f(\lambda)| v(E),
\end{aligned}
$$

where $v(E)$ is the constant of 3.2. Also if $\alpha$ is an arbitrary parameter and $f(\alpha, \lambda)$ is continuous for $\lambda \in \sigma(T)$ uniformly with respect to $\alpha$, then the sums $\sum_{\pi} f\left(\alpha, \lambda_{i}\right) E_{\Delta_{i}}$ for a partition $\pi=\left(\Delta_{i}, \ldots, \Delta_{n}\right), \lambda_{i} \in \Delta_{i}$, converge, as $|\pi| \longrightarrow 0$, uniformly with respect to $\alpha$.

For two partitions $\pi=\left(\Delta_{i}, \cdots, \Delta_{n}\right), \pi^{\prime}=\left(\Delta_{1}^{\prime}, \cdots, \Delta_{m}^{\prime}\right)$ of $\sigma(T)$, and for $\lambda_{i} \in \Delta_{i}, \lambda_{j}^{\prime} \in \Delta_{j}^{\prime}(i=1, \cdots, n, j=1, \cdots, m)$, we have

$$
\sum_{i=1}^{n} f\left(\alpha, \lambda_{i}\right) E_{\Delta_{i}}-\sum_{j=1}^{m} f\left(\alpha, \lambda_{j}^{\prime}\right) E_{\Delta_{j}^{\prime}}=\sum_{i=1}^{n} \sum_{j=1}^{m}\left\{f\left(\alpha, \lambda_{i}\right)-f\left(\alpha, \lambda_{j}^{\prime}\right)\right\} E_{\Delta_{i} \Delta_{j}^{\prime}}
$$


If for $\epsilon>0, \delta(\epsilon)>0$ is chosen so that

$$
\left|f(\alpha, \lambda)-f\left(\alpha, \lambda^{\prime}\right)\right|<\epsilon \text { for }\left|\lambda-\lambda^{\prime}\right|<\delta(\epsilon),
$$

we have, by 3.2 ,

$$
\begin{aligned}
& \left|\sum_{i=1}^{n} f\left(\alpha, \lambda_{i}\right) E_{\Delta_{i}}-\sum_{j=1}^{m} f\left(\alpha, \lambda_{j}^{\prime}\right) E_{\Delta_{j}}\right|<\epsilon v(E), \alpha,|\pi|,\left|\pi^{\prime}\right|<\delta(\epsilon) \\
& \left|\sum_{i=1}^{n} f\left(\alpha, \lambda_{i}\right) E_{\Delta_{i}}\right| \leq \max _{\lambda \in \sigma(T)}|f(\alpha, \lambda)| v(E),
\end{aligned}
$$

and

$$
\left|E_{\sigma} \sum_{i=1}^{n} f\left(\alpha, \lambda_{i}\right) E_{\Delta_{i}}\right| \leq \sup _{\lambda \in \sigma}|f(\alpha, \lambda)| v(E) ;
$$

this proves the lemma.

While in our final results the only operator-valued functions we shall have to integrate are of the form $(T-\lambda I)^{n} f(\lambda)$, where $f$ is a scalar function, it will, during the course of the proofs to follow, be necessary to integrate functions in a more extensive class. Accordingly, we consider functions of the following type. Let $D_{1}$ be an open set containing the closure $\bar{D}$ of an admissible open set $D \supset \sigma(T)$. Let $C$ be the boundary of $D$. Let $f(\alpha, \lambda)$ be a scalar function defined for $\alpha \in D_{1}, \lambda \in \sigma(T)$, continuous similtaneously in both variables over their respective ranges, and analytic for $\alpha \in D_{1}$ uniformly with respect to $\lambda \in \sigma(T)$; that is,

$$
\frac{f(\alpha+\eta, \lambda)-f(\alpha, \lambda)}{\eta} \rightarrow \frac{\partial f(\alpha, \lambda)}{\partial \alpha}
$$

uniformly with respect to $\lambda \in \sigma(T)$. Because the continuity in $\lambda$ is uniform with respect to $\alpha$ on $C$, the operator-valued function

$$
f(T, \lambda)=\frac{1}{2 \pi i} \int_{C} f(\alpha, \lambda) T(\alpha) d \alpha
$$

depends continuously on $\lambda$. It is this type of continuous operator-valued function defined by a scalar function $f(\alpha, \lambda)$ whose singularities in $\alpha$ stay uniformly 
away from $\sigma(T)$ as $\lambda$ varies over $\sigma(T)$ that we shall be integrating. For the sake of brevity we shall call functions $f(\alpha, \lambda)$ of the above type T-uniform.

3.4. Lemma. Let $f(\alpha, \lambda)$ be T-uniform. Then $f(T, \lambda)$ is integrable, and for every Borel set $\sigma$ we have

$$
\int_{\sigma} f(T, \lambda) d E_{\lambda}=\frac{1}{2 \pi i} \quad \int_{C}\left(\int_{\sigma} f(\alpha, \lambda) d E_{\lambda}\right) T(\alpha) d \alpha
$$

Let $\pi=\left(\Delta_{i}, \cdots, \Delta_{n}\right), \lambda_{i} \in \Delta_{i}$, be a partition of $\sigma(T)$. Then

$$
\begin{aligned}
\sum_{i} f\left(T, \lambda_{j}\right) E_{\Delta_{j}} & =\sum_{j}\left(\frac{1}{2 \pi i} \int_{C} f\left(\alpha, \lambda_{j}\right) T(\alpha) d \alpha\right) E_{\Delta_{j}} \\
& =\frac{1}{2 \pi i} \int_{C}\left(\sum_{j} f\left(\alpha, \lambda_{j}\right) E_{\Delta_{j}}\right) T(\alpha) d \alpha .
\end{aligned}
$$

The desired results follow from 3.3.

3.5. Lemma. Let $f(\alpha, \lambda), g(\alpha, \lambda)$ be T-uniform. Then $f(\alpha, \lambda) g(\alpha, \lambda)$ is T-uniform, and for a partition $\pi=\left(\Delta_{i}, \cdots, \dot{\Delta_{n}}\right)$ of $\sigma(T)$ and points $\lambda_{j}, \lambda_{j}^{\prime} \in \bar{\Delta}_{j}$ we have

$$
\lim _{|\pi| \rightarrow 0} \sum_{\pi} f\left(T, \lambda_{j}\right) g\left(T, \lambda_{j}^{\prime}\right) E_{\Delta_{j}}=\int f(T, \lambda) g(T, \lambda) d E_{\lambda}
$$

It is clear that there is a common domain of uniform analyticity. Let $C$ be its boundary. For $\epsilon>0$, fix $\delta>0$ such for every pair $\lambda, \lambda^{\prime} \in \sigma(T)$ with $\left|\lambda-\lambda^{\prime}\right|<\delta$ we have

$$
\left|f(\alpha, \lambda)\left[g\left(\alpha, \lambda^{\prime}\right)-g(\alpha, \lambda)\right]\right| \leq \epsilon, \alpha \in C .
$$

From 3.2, if $|\pi|<\delta$ then

$$
\sum_{\pi} f\left(\alpha, \lambda_{i}\right)\left[g\left(\alpha, \lambda_{j}^{\prime}\right)-g\left(\alpha, \lambda_{i}\right)\right] E_{\Delta_{j}} \mid \leq \epsilon v(E), \alpha \in C .
$$

Now

$$
f\left(T, \lambda_{j}\right) g\left(T, \lambda_{j}^{\prime}\right)=f\left(T, \lambda_{j}\right) g\left(T, \lambda_{j}\right)+f\left(T, \lambda_{j}\right)\left[g\left(T, \lambda_{j}^{\prime}\right]-g\left(T, \lambda_{j}\right)\right]
$$

and 


$$
\begin{aligned}
& \left|\sum f\left(T, \lambda_{j}\right)\left[g\left(T, \lambda_{j}^{\prime}\right)-g\left(T, \lambda_{j}\right)\right] E_{\Delta_{j}}\right| \\
& \quad=\left|\frac{1}{2 \pi i} \int_{C} T(\alpha) d \alpha\left(\sum f\left(\alpha, \lambda_{j}\right)\left[g\left(\alpha, \lambda_{j}^{\prime}\right)-g\left(\alpha, \lambda_{j}\right)\right] E_{\Delta_{j}}\right)\right| \\
& \left.\quad=(\epsilon / 2 \pi)\left(\max _{a \in C}|T(\alpha)|\right) \text { (length } C\right) v(E) .
\end{aligned}
$$

Thus in order to prove the lemma, it suffices to show that

$$
\lim _{\pi} \sum f\left(T, \lambda_{j}\right) g\left(T, \lambda_{j}\right) E_{\Delta_{j}}=\int f(T, \lambda) g(T, \lambda) d E_{\lambda}
$$

(Note that if $\lambda_{j} \in \Delta_{j}$ and not merely in $\bar{\Delta}_{j}$, there is nothing left to prove.) But this is clear since the function $f(T, \lambda) g(T, \lambda)$ is continuous in $\lambda$.

3.6. LeмmA. If $f$ is integrable (scaler-or operator-valued) and $U$ is a bounded linear operator in $X$ which commutes with $E_{e}$, $e \in B$, then $U f$ is integrable and

$$
U \int_{\sigma} f(\lambda) d E_{\lambda}=\int_{\sigma} U f(\lambda) d E_{\lambda}, \sigma \in B
$$

The proof is clear from the definitions.

3.7. TheOREM. Let $X$ be arbitrary and $T$ a bounded linear operator in $X$ with the resolution of the identity $E_{e}$. For any closed set of points $\rho \in \rho(T)$, we have

$$
(\xi-T)^{-1}=\sum_{n=0}^{\infty} \int \frac{(T-\lambda)^{n}}{(\xi-\lambda)^{n+1}} d E_{\lambda},
$$

where the sum converges in the uniform topology of operators and uniformly with respect to $\xi \in \rho$.

In view of the elementary identity

$$
(\xi-T) \sum_{n=0}^{p} \frac{(T-\lambda)^{n}}{(\xi-\lambda)^{n+1}}=I-\frac{(T-\lambda)^{p+1}}{(\xi-\lambda)^{p+1}}
$$

and 3.6, we have 


$$
(\xi-T) \sum_{n=0}^{p} \frac{(T-\lambda)^{n}}{(\xi-\lambda)^{n+1}} d E_{\lambda}=I-\int \frac{(T-\lambda)^{p+1}}{(\xi-\lambda)^{p+1}} d E_{\lambda}
$$

Now let $\delta=$ the distance from $\rho$ to $\sigma(T)$. This is positive since $\sigma(T)$ is bounded and closed. Break $\sigma(T)$ into disjoint measurable parts $\Delta_{1}, \ldots, \Delta_{n}$ of which the diameters satisfy

$$
\text { diameter } \Delta_{j} \leq \delta / 4
$$$$
(j=1, \cdots, n) \text {. }
$$

Let $C_{j}$ be a circle of diameter $\delta / 2$ containing $\bar{\Delta}_{j}$ in its interior, so that

$$
\left|(\alpha-\lambda)(\xi-\lambda)^{-1}\right| \leq 1 / 2, \xi \in \rho, \lambda \in \Delta_{j}, \quad \alpha \in C_{j}
$$

Let $T_{\alpha}\left(\Delta_{j}\right)$ be the resolvent of $T$ when considered as an operator in $X_{\Delta_{j}}$. Since $\sigma\left(X_{\bar{\Delta}_{j}}\right) \subset \bar{\Delta}_{j}$ and

$$
\left(\int_{\Delta_{j}} \frac{(T-\lambda)^{p}}{(\xi-\lambda)^{p}} d E_{\lambda}\right) X \subset X_{\bar{\Delta}_{j}}
$$

we have, from 3.3,

$$
\begin{aligned}
& \left|\int_{\Delta_{j}} \frac{(T-\lambda)^{p}}{(\xi-\lambda)^{p}} d E_{\lambda}\right| \\
& =\mid \int_{\Delta_{j}}\left(\frac{1}{2 \pi i} \int_{C_{j}} \frac{(\alpha-\lambda)^{p}}{(\xi-\lambda)^{p}} T_{\alpha}\left(\Delta_{j}\right) d \alpha\left|d E_{\lambda}\right|\right. \\
& =\left|\frac{1}{2 \pi i} \int_{C_{j}} T_{\alpha}\left(\Delta_{j}\right) d \alpha \int_{\Delta_{j}} \frac{(\alpha-\lambda)^{p}}{(\xi-\lambda)^{p}} d E_{\lambda}\right| \\
& \leq \frac{1}{2 \pi} \max _{\substack{a \in C_{j}\\
}}\left|T_{\alpha}\left(\Delta_{j}\right)\right| v(E) / 2^{p}, \quad \xi \in \rho .
\end{aligned}
$$

Since $\int=\int_{\Delta_{1}}+\cdots+\int_{\Delta_{n}}$, we have

$$
(\xi-T) \sum_{n=0}^{p} \int \frac{(T-\lambda)^{n}}{(\xi-\lambda)^{n+1}} d E_{\lambda} \rightarrow I
$$


uniformly for $\xi \in \rho$. This proves the theorem.

3.8. DEFINITION. An operator is called a spectral operator if it has a resolution of the identity.

3.9. THEOREM. If $T$ is a bounded spectral operator, the resolution of the the identity $E_{e}$ is unique, and for every $f \in F(T)$ we have

$$
f(T)=\sum_{n=0}^{\infty} \int \frac{f^{(n)}(\lambda)}{n !}(T-\lambda)^{n} d E_{\lambda},
$$

where the integral exists as a Riemann integral in the uniform topology of operators and the sum converges in the uniform topology of operators.

We shall first show uniqueness. Let $A_{e}, E_{e}$ be resolutions of the identity for $T$. Let $\sigma_{1}, \sigma_{2}$ be disjoint and each consist of a circle and its interior. Let $T^{1}(\xi)$ be the resolvent of $T$ when considered as an operator on $E_{\sigma_{1}} X$. Let $T^{2}(\xi)$ be the resolvent of $T$ when considered as an operator in $A_{\sigma_{2}} X$. Then for $\xi \notin \sigma_{1}, T^{1}(\xi) E_{\sigma_{1}} A_{\sigma_{2}}$ is a bounded linear operator in $X$ and analytic for $\xi \in \sigma_{1}^{\prime}$. Likewise for $\xi \in \sigma_{2}^{\prime}, E_{\sigma_{1}} T^{2}(\xi) A_{\sigma_{2}}$ is a bounded linear operator in $X$ and analytic for $\xi \in \sigma_{2}^{\prime}$. Let $\xi \in\left(\sigma_{1} \cup \sigma_{2}\right)^{\prime}$. Since $\xi-T$ commutes with $E_{\sigma_{1}}$, we have

$$
(\xi-T) E_{\sigma_{1}} T^{2}(\xi) A_{\sigma_{2}}=E_{\sigma_{1}} A_{\sigma_{2}},
$$

and operating on the left with $T^{1}(\xi)$ we have

$$
E_{\sigma_{1}} T^{2}(\xi) A_{\sigma_{2}}=T^{\prime}(\xi) E_{\sigma_{1}} A_{\sigma_{2}} \cdot
$$

If $f(\xi)$ is defined to be

$$
\begin{aligned}
f(\xi) & =E_{\sigma_{1}} T^{2}(\xi) A_{\sigma_{2}}=T^{1}(\xi) E_{\sigma_{1}} A_{\sigma_{2}}, \xi \in\left(\sigma_{1} \cup \sigma_{2}\right)^{\circ} \\
& =E_{\sigma_{1}} T^{2}(\xi) A_{\sigma_{2}}, \quad \xi \in \sigma_{1} \\
& =T^{1}(\xi) E_{\sigma_{1}} A_{\sigma_{2}}, \quad \xi \in \sigma_{2},
\end{aligned}
$$

then $f$ is an entire function. Since for large $\xi$, we have

$$
f(\xi)=T(\xi) E_{\sigma_{1}} A_{\sigma_{2}} \rightarrow 0 \quad \text { as } \quad|\xi| \rightarrow \infty \text {. }
$$


it follows that $f(\xi)=0$ and $E_{\sigma_{1}} A_{\sigma_{2}}=0$. By symmetry, $A_{\sigma_{2}} E_{\sigma_{1}}=0$. Let $\sigma_{n}$, $(n=3,4, \cdots)$ be the set of those points of $\sigma(T)$ whose distance from $\sigma_{1}$ is $\geq 1 / n$. Let $\delta_{1}, \cdots, \delta_{p(n)}$ each consist of a circle and its interior and be such that the set $\delta^{n}=\mathrm{U} \delta_{i}$ is disjoint with $\sigma_{1}$ and covers $\sigma_{n}$. Then, since $E_{\sigma_{1}} A_{\delta_{i}}=0$, we have $A_{\delta_{i}} \subset E_{\sigma_{1}^{\prime}} X$ and

$$
A_{\sigma_{n}} X \subset A_{\delta^{n}} X=\left(\underset{i}{\cup} A_{\delta_{i}}\right) X=\underset{i}{\bigcup}\left(A_{\delta_{i}} X\right) \subset E_{\sigma_{1}} X
$$

Hence

$$
E_{\sigma_{1}}^{\prime} A_{\sigma_{n}}=A_{\sigma_{n}}, E_{\sigma_{1}} A_{\sigma_{n}}=0
$$

But $A_{\sigma_{n}} x \rightarrow A_{\sigma_{1}^{\prime}} x, x \in X$, and so

$$
E_{\sigma_{1}} A_{\sigma_{1}}^{\prime}=0, E_{\sigma_{1}} A_{\sigma_{1}}=E_{\sigma_{1}}
$$

Also, since $A_{\delta_{i}} E_{\sigma_{1}}=0$, we have $E_{\sigma_{1}} X \subset A_{\delta_{i}^{\prime}} X$ and

$$
E_{\sigma_{1}} X \subset \bigcap_{i}\left(A_{\delta_{i}^{\prime}} X\right)=\left(\bigcap_{i} A_{\delta_{i}^{\prime}}\right) X=A_{\delta^{n}}^{\prime} X .
$$

Thus

$$
A_{\delta^{n}}^{\prime} E_{\sigma_{1}}=E_{\sigma_{1}}, A_{\delta^{n}} E_{\sigma_{1}}=0, A_{\sigma_{1}}^{\prime} E_{\sigma_{1}}=0, A_{\sigma_{1}} E_{\sigma_{1}}=E_{\sigma_{1}},
$$

and therefore

$$
E_{\sigma_{1}}=A_{\sigma_{1}} E_{\sigma_{1}}=E_{\sigma_{1}} A_{\sigma_{1}}=E_{\sigma_{1}} .
$$

By symmetry,

$$
A_{\sigma_{1}}=A_{\sigma_{1}} E_{\sigma_{1}}=E_{\sigma_{1}} A_{\sigma_{1}}=E_{\sigma_{1}}
$$

From this it readily follows that $A_{\sigma}=E_{\sigma}$ for any Borel set $\sigma$. Now let $f \in F(T)$. Let $C$ be an admissible contour upon which $f$ is analytic and such that

$$
f(T)=\frac{1}{2 \pi i} \int_{C} f(\xi) T(\xi) d \xi
$$

Now, by 3.7, we have 


$$
T(\xi)=\sum_{n=0}^{\infty} \int(T-\lambda)^{n}(\xi-\lambda)^{-n-1} d E_{\lambda},
$$

and the series converges in the uniform topology of operators and uniformly for $\xi \in C$. So

$$
f(T)=\sum_{n=0}^{\infty} \frac{1}{2 \pi i} \int_{C} f(\xi) d \xi\left(\int \frac{(T-\lambda)^{n}}{(\xi-\lambda)^{n+1}} d E_{\lambda}\right) .
$$

Since

$$
\int \frac{(T-\lambda)^{n}}{(\xi-\lambda)^{n+1}} d E_{\lambda}=\sum_{n=0}^{n}\left(\begin{array}{l}
n \\
r
\end{array}\right) T^{r} \int \frac{\lambda^{(n-r)}}{(\xi-\lambda)^{n+1}} d E_{\lambda},
$$

it follows from 3.3 , that the Riemann sums approximating

$$
\int(T-\lambda)^{n}(\xi-\lambda)^{-n-1} d E_{\lambda}
$$

converge uniformly with respect to $\xi \in C$.

Hence

$$
f(T)=\sum_{n=0}^{\infty} \int \frac{f^{(n)}(\lambda)}{n !}(T-\lambda)^{n} d E_{\lambda} .
$$

From this point on we shall again restrict our attention to the case of an operator $T$ whose spectrum lies in a rectifiable Jordan curve $\Gamma_{0}$ and whose resolvent satisfies the growth condition 2.1. It will be convenient to state the condition 1.14 in terms of residues as defined in the following:

3.10. DEFINITION. Let $\sigma(x), \sigma\left(x^{*} x\right)$ be the sets of singularities of the functions $x(\xi)=(\xi-T)^{-1} x, x^{*} x(\xi)$, respectively. Let $\sigma$ be open and closed in $\sigma(x)$, and

$$
x_{\sigma}=\frac{1}{2 \pi i} \quad \int_{C} x(\xi) d \xi
$$

where $C$ is a rectifiable Jordan curve containing $\sigma$ in its interior and having $\sigma(x) \sigma^{\prime}$ in its exterior. Then the vector $x_{\sigma}$ is called the $\sigma$-residue of $x(\xi)$. 
Similarly if $\sigma$ is open and closed in $\sigma\left(x^{*} x\right)$ then the scalar

$$
\left(x^{*} x\right)_{\sigma}=\frac{1}{2 \pi i} \int_{C} x^{*} x(\xi) d \xi
$$

is called the $\sigma$-residue of $x^{*} x(\xi)$.

With this terminology, condition 1.14 asserts the existence of a constant $K$ such that $\left|x_{\sigma}\right| \leq K|x|$.

3.11. THE OREM. Let $X$ be weakly complete, and let $T$ be a bounded linear operator in $X$ whose spectrum lies in the rectifiable Jordan curve $\Gamma_{0}$ and whose resolvent is restricted in its growth by Assumption 2.1. Then $T$ is a spectral operator and satisfies the equation

$$
f(T)=\sum_{n=0}^{\infty} \int_{\sigma(T)} \frac{f^{(n)}(\xi)}{n !}(T-\xi)^{n} d E_{\xi}, f \in F(T),
$$

providing:

(i) (The density condition.) For every $\xi$ in a set dense on $\Gamma_{0} \mathbb{M}_{\xi}+\Re_{\xi}$ is dense in $X$.

(ii) (The boundedness condition.) There is a constant $K$ such that all residues $x_{\sigma}$ satisfy the inequality

$$
\left|x_{\sigma}\right| \leq K|x|, x \in X
$$

This theorem is an immediate corollary of 2.11 and 3.8.

Conditions will now be given which are of a nature more applicable than (i) and (ii) of 3.11 and which are sufficient (and in some cases necessary) to imply (i) and (ii). We shall begin with a brief analysis of some conditions which are sufficient to imply the density condition (i).

3.12. THEOREM. The operator $T$ of Theorem 3.11 satisfies the density condition (i) of that theorem in case any one of the following is true:

(i) Every subarc (of positive length) of $\Gamma_{0}$ contains points either in the continuous spectrum or in the resolvent set.

(ii) No subarc (of positive length) of $\Gamma_{0}$ consists entirely of points in the 
point spectrum of the adjoint $T^{*}$ of $T$.

(iii) The space $X$ is reflexive and $\nu(\lambda)=1$ is an index function for $T$.

(iv) The space $X$ is reflexive and the adjoint $T^{*}$ satisfies the boundedness condition (ii) of 3.11 .

(v) The operator $T$ is completely continuous.

The first statement is obvious since if $\xi$ is in either the resolvent set or the continuous spectrum we have $\Re_{\xi}=X$. The second statement is equally clear since it is seen from the Hahn-Banach theorem that $\xi$ is in the point spectrum of the adjoint if and only if $\Re_{\xi} \neq X$. Next, if (iii) holds, let

$$
\xi=(\lambda, \delta), \xi_{0}=\xi(\lambda, 0),
$$

so that

$$
\left|\left(\xi-\xi_{0}\right) T(\xi)\right| \leq|\delta T(\xi)| \leq 1 .
$$

Now

$$
\left(\xi-\xi_{0}\right) T(\xi)\left(\xi_{0}-T\right)=\xi-\xi_{0}-\left(\xi-\xi_{0}\right)^{2} T(\xi),
$$

and hence

$$
\lim _{\delta \rightarrow 0}\left(\xi-\xi_{0}\right) T(\xi)\left(\xi_{0}-T\right)=0
$$

Now let $x$ be an arbitrary vector in $X$. Since $X$ is reflexive, the set

$$
\left(\xi-\xi_{0}\right) T(\xi) x, \quad 0<\delta \leq \delta_{0}
$$

is weakly compact, and there is a vector $y \in X$ and a sequence $\delta_{n} \longrightarrow 0$ such that for $\xi_{n}=\xi\left(\lambda, \delta_{n}\right)$ we have

$$
\left(\xi_{n}-\xi_{0}\right) T\left(\xi_{n}\right) x \rightarrow y \text { weakly }
$$

The equation $(*)$ shows that $y \in \mathbb{N}_{\xi_{0}}$. To see that $x-y \in \Re_{\xi_{0}}$, let $x * \Re_{\xi_{0}}=0$. Then

$$
x^{*}\left(\xi-\xi_{0}\right) T(\xi)=x^{*}
$$


and so $x^{*}(x-y)=0$. Hence $\Re_{\xi_{0}}+\Re_{\xi_{0}}=X$. To prove the fourth statement we note first that, since $\sigma(T)=\sigma\left(T^{*}\right)$ and $|T(\xi)|=\left|T^{*}(\xi)\right|$, the adjoint $T^{*}$ satisfies 2.1, and any index function $\nu(\lambda)$ for $T$ is also an index function for $T^{*}$. By 2.9, then, $\mathbb{M}_{\xi}\left(T^{*}\right) \Re_{\xi}\left(T^{*}\right)=0$ for $\xi$ interior to any interval upon which an index function is constant. Such $\xi$ are by 2.3 dense on $\Gamma_{0}$. Let $\xi$ be such a point on $\Gamma_{0}$, and let

$$
x * M_{\xi}=0=* * \Omega_{\xi}
$$

It will suffice to prove that $x^{*}=0$. Since $x^{*} \Re_{\xi}=0$, we have $x^{*} \in \mathbb{M}_{\xi}\left(T^{*}\right)$. To see that $x^{*} \in \Re_{\xi}\left(T^{*}\right.$ ) (which will prove $x^{*}=0$ ), it will suffice, since $X$ is reflexive, to show that $x^{*} x_{0}=0$ for every $x_{0}$ with $\Re_{\xi}\left(T^{*}\right) x_{0}=0$, that is, for every $x_{0}$ with

$$
y^{*}(\xi-T)^{\nu} x_{0}=0, y^{*} \in Y^{*}
$$

But such an $x_{0}$ is in $M_{\xi}$, and so $x^{*} x_{0}=0$. The final statement $(\mathrm{v})$ follows from the fact that the spectrum of a completely continuous operator is at most denumerable.

N. B. As the above proof shows, the condition that $X$ be reflexive (in (iv)) may be replaced by the statement that, for $\xi$ in a dense set on $\Gamma_{0}$, the manifold $\Re_{\xi}\left(T^{*}\right)$ is regularly closed. Also in (iii) the condition of reflexivity may be replaced by the assumption that the set of vectors $\left(\xi-\xi_{0}\right) T(\xi) x, 0<\delta<\delta_{0}$, is weakly compact.

3.13. THEOREM. Let $X$ be a reflexive space and $T$ a bounded linear operator in $X$ whose spectrum lies in the rectifiable Jordan curve $\Gamma_{0}$ and whose resolvent satisfies the growth condition 2.1. Then $T$ is a spectral operator and satisfies the equation

$$
f(T)=\sum_{n=0}^{\infty} \int_{\sigma(T)} \frac{f^{(n)}(\xi)}{n !}(T-\xi)^{n} d E_{\xi}, f \in F(T),
$$

if and only if there is a constant $K$ such that all the residues $\left(x^{*} x\right)_{\sigma}$ satisfy the inequality.

$$
\left|\left(x^{*}, x\right)_{\sigma}\right| \leq K|x|\left|x^{*}\right|
$$

The residue condition is clearly necessary since 


$$
\left(x^{*} x\right)_{\sigma}=x^{*} E_{\sigma} x
$$

To see that it is sufficient we note first that it implies the condition (ii) of Theorem 3.11. Since $X$ is reflexive, the residue $\left(x^{*}, x\right)_{\sigma}$ is equal to the residue $\left(x^{* *}, x^{*}\right)_{\sigma}$ calculated for the adjoint $T^{*}$ (here $x^{* *} x^{*}=x^{*} x, x^{*} \in X^{*}$ ) and so the residue condition of 3.13 implies that the adjoint $T^{*}$ satisfies (ii) of 3.11 . The present theorem then follows from 3.11 and 3.12 (iv).

We now turn our attention to stating the requirement (of 3.11 or 3.13 ) that the residues be bounded, in a form which, in some instances, is more readily applied.

3.14. THЕ овем. Let $\xi=\xi(\lambda, \delta)$ have continuous first partial derivatives, and let $\xi^{-}=\xi(\lambda,-\delta)$. Then the residues $\left(x^{*}, x\right)_{\sigma}$ and $x_{\sigma}$ will have a bound of the form $K|x|\left|x^{*}\right|$ in case

$$
\underset{0<\delta<\delta_{0}}{\text { l.u.b. }} \int_{-1}^{+1}\left|x^{*}\left\{T(\xi) \frac{\partial \xi}{\partial \lambda}-T\left(\xi^{-}\right) \frac{\partial \xi^{-}}{\partial \lambda}\right\} x\right| d \lambda \leq M|x|\left|x^{*}\right| \text {. }
$$

Let $0 \leq \lambda_{j}<\lambda_{j}^{\prime} \leq 1$, let $C_{j}=C\left(\lambda_{j}, \lambda_{j}^{\prime}\right)$ be as in 2.4 , and let $C$ be the set $C_{j}, \quad(j=1, \cdots n)$. Suppose that $C$ lies in the domain of analyticity of $x^{*} x(\xi)$. Then

$$
\int_{C} x^{*} x(\xi) d \xi=\sum_{j=1}^{n} \int_{\lambda_{j}}^{\lambda_{j}^{\prime}} x^{*}\left\{x(\xi) \frac{\partial \xi}{\partial \lambda}-x\left(\xi^{-}\right) \frac{\partial \xi^{-}}{\partial \lambda}\right\} d \lambda+I(\delta)
$$

where $I(\delta)$ is a sum of integrals $\int x^{*} x(\xi) d \xi$ taken along the arcs $\xi\left(\lambda_{j}, \mu\right)$, $\xi\left(\lambda_{j}^{\prime}, \mu\right),-\delta \leq \mu \leq \delta$. Thus $\lim _{\delta \rightarrow 0} I(\delta)=0$, and

$$
\begin{aligned}
& \limsup _{\delta \rightarrow 0}\left|\int_{C} x^{*} x(\xi) d \xi\right| \\
& \leq \underset{0<\delta<\delta_{0}}{\text { l.u.b. }} \int_{-1}^{+1}\left|x^{*}\left\{T(\xi) \frac{\partial \xi}{\partial \lambda}-T\left(\xi^{-}\right) \frac{\partial \xi}{\partial \lambda}\right\} x\right| d \lambda \leq M|x|\left|x^{*}\right|
\end{aligned}
$$

The condition of 3.13 is far from necessary, and is not satisfied by the resolvent $T(\xi)$ if its rate of growth for $\xi$ near $\sigma(T)$ is not that of the inverse of the distance from $\xi$ to $\sigma(T)$. To avoid this objection a similar condition, as is evident from the above proof, may be stated. 
3.15. TheоREM. Let $T(\xi)=U(\xi)+V(\xi)$, where $x^{*} V(\xi) x$ is the derivative of a single valued analytic function at each point $\xi$ where $x^{*} T(\xi) x$ is analytic. Then the residues $\left(x^{*}, x^{*}\right)_{\sigma}$ and $x_{\sigma}$ will have a bound of the forms $K|x|\left|x^{*}\right|$ provided that $U(\xi)$ satisfies the condition of 3.14 .

\section{Operators of finite type}

As is to be expected from the analogy with the elementary divisor theory for a finite matrix, certain spectral operators should satisfy the formula

$$
f(T)=\sum_{n=0}^{m-1} \int_{\sigma(T)} \frac{f^{(n)}(\xi)}{n !}(T-\xi)^{n} d E_{\xi}, f \in F(T) .
$$

One might expect this to be true if the spectrum $\sigma(T)$ is nowhere dense and if the resolvent $T(\xi)$ has for $\xi$ near $\sigma(T)$ the same rate of growth as

$$
\left[\operatorname{dis}(\xi, \sigma(T)]^{-m}\right.
$$

We have been able to prove this only in the case where $\sigma(T)$ is restricted to lie in a sufficiently smooth Jordan curve.

We shall assume throughout the following discussion that the function $\xi(\lambda, \mu)$ defining the net described in 2.1 has continuous second partial derivatives. The purpose of this assumption is to assure that the length of the contour $C\left(\lambda_{1}, \lambda_{2}\right)$ of 2.4 is at most $K \delta$, provided that $\lambda_{1}<\lambda_{2}$ and $\delta=\lambda_{2}-\lambda_{1}$. Also the diameter of $C\left(\lambda_{1}, \lambda_{2}\right)$ is at most $K \delta$ for $\delta=\lambda_{2}-\lambda_{1}$.

3.16. LеммA. (Assumption 2.1.) Let $d(\xi)$ be the distance from $\xi$ to the spectrum $\sigma(T)$. If $\left|d^{m}(\xi) T(\xi)\right|$ is bounded for $\xi$ near $\sigma(T)$, then

$$
\int_{\sigma(T)} f(T, \xi)(T-\xi)^{2 m} d E_{\xi}=0
$$

for every T-uniform $f(\alpha, \xi)$.

We may and shall assume that $\nu(\lambda)=m$ is an index function for $T$, so that

$$
\left|\delta^{m} T_{(\xi)}\right| \leq 1,0<|\delta|<\delta_{0}, \lambda \in[-1,1]
$$

Let $\lambda_{1}<\lambda_{2}, \lambda_{2}-\lambda_{1}<\delta_{0}$. Let $C\left(\lambda_{1}, \lambda_{2}\right)$ be the contour defined in 2.4 with $\delta=\lambda_{2}-\lambda_{1}$. Let $\Delta$ be the closed subarc of $\Gamma_{0}$ defined by $\xi(\lambda, 0), \lambda_{1} \leq \lambda \leq \lambda_{2}$. 
Let $I\left(\lambda_{1}, \lambda_{2}\right)$ be the integral defined in 2.4 with $\nu_{1}=\nu_{2}=m$ and $P(\xi)=1$. Let $\lambda_{n}<\lambda_{1}, \lambda_{2}<\mu_{n}, \lambda_{n} \rightarrow \lambda_{1}, \mu_{n} \rightarrow \lambda_{2}$. By 2.4 , we have $I\left(\lambda_{n}, \lambda_{1}\right) \rightarrow 0$, $I\left(\lambda_{2}, \mu_{n}\right) \longrightarrow 0$. Also, by $2.4,1.39$ (ii), 2.5, and 1.12, we have $E_{\Delta} I\left(\mu_{n}, \lambda_{n}\right)=0$. If $\xi_{j}=\xi\left(\lambda_{j}, 0\right)(j=1,2)$, then

$$
\left(T-\xi_{1}\right)^{m}\left(T-\xi_{2}\right)^{m}=I\left(\lambda_{n}, \lambda_{1}\right)+I\left(\lambda_{1}, \lambda_{2}\right)+I\left(\lambda_{2}, \mu_{n}\right)+I\left(\mu_{n}, \lambda_{n}\right) ;
$$

and so we have

$$
E_{\Delta}\left(T-\xi_{1}\right)^{m}\left(T-\xi_{2}\right)^{m}=E_{\Delta} I\left(\lambda_{1}, \lambda_{2}\right)
$$

But by 2.4, we have $\sigma\left(I\left(\lambda_{1}, \lambda_{2}\right) x\right) \subset \Delta$; hence by 1.39 (i) it is seen that $I\left(\lambda_{1}, \lambda_{2}\right)=E_{\Delta} I\left(\lambda_{1}, \lambda_{2}\right)$. Thus

$$
E_{\Delta}\left(T-\xi_{1}\right)^{m}\left(T-\xi_{2}\right)^{m}=I\left(\lambda_{1}, \lambda_{2}\right) .
$$

Now, since $\delta=\lambda_{2}-\lambda_{1}$, there are constants $K_{1}, K_{2}$ such that

$$
\max _{\lambda_{1} \leq \lambda \leq \lambda_{2}}\left|\xi(\lambda, \pm \delta)-\xi\left(\lambda_{1}, 0\right)\right| \leq K_{1} \delta
$$

and

$$
\text { length } C\left(\lambda_{1}, \lambda_{2}\right) \leq K_{2} \delta \text {. }
$$

It follows from the definition of $I\left(\lambda_{1}, \lambda_{2}\right)$ therefore that

$$
\left|I\left(\lambda_{1}, \lambda_{2}\right)\right| \leq K_{3} \delta^{m+1}
$$

Let the interval $[-1,1]$ be partitioned into $n$ intervals $\left[\lambda_{j-1}, \lambda_{j}\right]$ each of length $2 / n$, and let $\Delta_{j}$ be the corresponding subarcs of $\Gamma_{0}$ with end points $\xi_{j-1}, \xi_{j}$. Statements $(*)$ and $(\dagger)$ then give

$$
\left|\sum_{j=1}^{n} f\left(T, \xi_{j}\right)\left(T-\xi_{j}\right)^{m}\left(T-\xi_{j-1}\right)^{m} E_{\Delta_{j}}\right| \leq K_{4} n^{-m} .
$$

Hence, by 3.5 , we have

$$
\int_{\sigma(T)} F(T, \xi)(T-\xi)^{2 m} d E_{\xi}=0
$$


3.17. LemMA. Under the hypothesis of 3.16, we have

$$
(T-\xi)^{m} E_{(\xi)}=0,
$$

where $(\xi)$ is the set consisting of the single point $\xi$.

From 3.16, we get

$$
(T-\xi)^{2 m} E_{(\xi)}=E_{(\xi)} \quad \int_{\sigma(T)}(T-\mu)^{2 m} d E_{\mu}=0
$$

Thus

$$
T(\alpha) E_{(\xi)}=\sum_{j=0}^{2 m-1} \frac{(T-\xi)^{j} E}{(\alpha-\xi)^{j+1}}(\xi)
$$

has a pole of order $\leq 2 m$ at $\alpha=\xi$. Since $\left|d^{m}(\alpha) T(\alpha)\right|$ is bounded for $\alpha$ near $\xi$, the pole must be of order $\leq m$; that is, $(T-\xi)^{m} E_{(\xi)}=0$.

3.18. LEMMA. Under the hypothesis of 3.16, we have

$$
\int_{\sigma(T)} f(T, \xi)(T-\xi)^{j} d E_{\xi}=0, j \geq m
$$

for every T-uniform function $f(\alpha, \xi)$.

$$
\begin{gathered}
\text { For } \xi=\xi(\lambda, 0) \in \Gamma_{0} \text { and } 0<|\delta|<\delta_{0} \text {, let } \xi_{\delta}=\xi(\lambda, \delta) \text {. Then } \\
(\xi-T) T\left(\xi_{\delta}\right)=\left(\xi-\xi_{\delta}\right) T\left(\xi_{\delta}\right)+I .
\end{gathered}
$$

Now assume for the purposes of induction (the above equality is the case $j=1$,) that

$$
\begin{aligned}
(\xi-T)^{j} T\left(\xi_{\delta}\right)=\left(\xi-\xi_{\delta}\right)^{j} T\left(\xi_{\delta}\right) & +\left(\xi-\xi_{\delta}\right)^{j-1}+\left(\xi-\xi_{\delta}\right)^{j-2}(\xi-T) \\
& +\cdots+\left(\xi-\xi_{\delta}\right)(\xi-T)^{j-2}+(\xi-T)^{j-1}
\end{aligned}
$$

Multiplying by $(\xi-T)$, we have 


$$
\begin{aligned}
&(\xi-T)^{j+1} T\left(\xi_{\delta}\right)=(\xi-T)^{j}(\xi-T) T\left(\xi_{\delta}\right)=(\xi-T)^{j}\left[\left(\xi-\xi_{\delta}\right) T\left(\xi_{\delta}\right)+I\right] \\
&=\left(\xi-\xi_{\delta}\right)^{j+1} T\left(\xi_{\delta}\right)+\left(\xi-\xi_{\delta}\right)^{j}+\left(\xi-\xi_{\delta}\right)^{j-1}(\xi-T) \\
&+\cdots+\left(\xi-\xi_{\delta}\right)(\xi-T)^{j-1}+(\xi-T)^{j}
\end{aligned}
$$

\section{Hence}

$$
\begin{aligned}
& f(T, \xi)\left[(\xi-T)^{j+1} T\left(\xi_{\delta}\right)-(\xi-T)^{j}\right] \\
& \quad=f(T, \xi)\left[\left(\xi-\xi_{\delta}\right)^{j+1} T\left(\xi_{\delta}\right)+\left(\xi-\xi_{\delta}\right)^{j}+\cdots+\left(\xi-\xi_{\delta}\right)(\xi-T)^{j-1}\right] .
\end{aligned}
$$

Thus we may state:

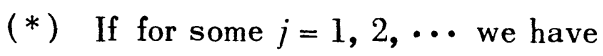

$$
\int_{\sigma(T)} f(T, \xi)(\xi-T)^{j+1} T\left(\xi_{\delta}\right) d E_{\xi}=0,0<|\delta|<\delta_{0},
$$

then

$$
\lim _{\delta \rightarrow 0} \int_{\sigma(T)} f(T, \xi)\left(\xi-\xi_{\delta}\right)^{j+1} T\left(\xi_{\delta}\right) d E_{\xi}=-\int_{\sigma(T)} f(T, \xi)(\xi-T)^{j} d E_{\xi} \cdot
$$

Now let $0<\left|\delta_{i}\right|<\delta_{0},(i=1,2, \cdots, m)$. By 3.16, then,

$$
\int_{\sigma(T)} f(T, \xi)(T-\xi)^{2 m} T\left(\xi_{\delta_{1}}\right) T\left(\xi_{\delta_{2}}\right) \cdots T\left(\xi_{\delta_{m}}\right) d E_{\xi}=0
$$

To this equation we may apply $(*)$ with $\delta=\delta_{1}$, and with $f(T, \xi)$ replaced by $f(T, \xi) T\left(\xi_{\delta_{2}}\right) \cdots T\left(\xi_{\delta_{m}}\right)$. Thus,

$$
\begin{aligned}
\lim _{\delta_{1} \rightarrow 0} \int_{\sigma(T)} f(T, \xi) & \left(\xi-\xi_{\delta_{1}}\right)^{2 m} T\left(\xi_{\delta_{1}}\right) \cdots T\left(\xi_{\delta_{m}}\right) d E_{\xi} \\
= & -\int_{\sigma(T)} f(T, \xi)(\xi-T)^{2 m-1} T\left(\xi_{\delta_{2}}\right) \cdots T\left(\xi_{\delta_{m}}\right) d E_{\xi}
\end{aligned}
$$

Since

$$
\left|\left(\xi-\xi_{\delta_{1}}\right)^{2 m} T\left(\xi_{\delta_{1}}\right)\right| \leq K\left|\delta_{1}\right|^{m},
$$


the integrand on the right side of the preceeding equation approaches zero with $\delta_{1}$ and uniformly with respect to $\xi \in \sigma(T)$. Thus

$$
\int_{\sigma(T)} f(T, \xi)(\xi-T)^{2 m-1} T\left(\xi_{\delta_{2}}\right) \cdots T\left(\xi_{\delta_{m}}\right) d E_{\xi}=0
$$

A repetition of this process clearly yields the desired result:

$$
\int_{\sigma(T)} f(T, \xi)(\xi-T)^{j} d E_{\xi}=0, \quad j \geq m
$$

3.19. Definition. Let $m$ be a positive integer. A spectral operator $T$ is said to be of type $m$ in case

$$
f(T)=\sum_{n=0}^{m-1} \int_{\sigma(T)} \frac{f^{(n)}(\xi)}{n !}(T-\xi)^{n} d E_{\xi}
$$

for every $f$ single valued and analytic on $\sigma(T)$, that is, for $f \in F(T)$.

Let us recall that for the case in hand (that is, $\nu(\lambda)=m$ is an index function), the manifolds $\mathbb{M}_{\xi}$, $\Re_{\xi}$ are respectively the zeros and the closure of the range $(T-\xi)^{m}$. Then if $d(\xi)$ is the distance from $\xi$ to the spectrum $\sigma(T)$ we may state:

3.20. THE OREM. If $X$ is weakly complete, $T$ will be a spectral operator and of type $m$ providing

(i) $\quad d^{m}(\xi) T(\xi)$ is bounded for $\xi$ near $\sigma(T)$,

(ii) for $\xi$ in a set dense in $\Gamma_{0}$ the manifold $s \Omega_{\xi}+\Omega_{\xi}$ is dense in $X$.

(iii) all residues $x_{\sigma}$ have a bound of the form $K|x|$.

This theorem follows immediately from 3.11 and 3.18.

N. B. 1. As before, the condition (ii) is automatically satisfied if $T$ enjoys any one of the properties listed in 3.12. Also (iii) is satisfied if the resolvent $T(\xi)$ satisfies the mean rate of growth condition of 3.14 or 3.15 .

N. B. 2. In case $X$ is not weakly complete it is still true that $E_{\Delta}$ is defined for every closed subarc of $\Gamma_{0}$ (see proof of 2.11 ), and $E_{\Delta}$ is completely additive, in the strong topology of operators, on the Boolean algebra determined by such arcs. Thus the integral 


$$
\int_{\sigma(T)} \frac{f^{(n)}(\xi)}{n !}(T-\xi)^{n} d E_{\xi}
$$

may be defined and the operational calculus developed even though $E_{e}$ may not be defined as an operator in $X$ for every Borel set $e$.

An immediate corollary is ( see 3.13$)$ :

3.21. THЕОВЕМ. If $X$ is reflexive, then $T$ will be a spectral operator of type $m$ if and only if

(i) $d^{m}(\xi) T(\xi)$ is bounded for $\xi$ near $\sigma(T)$,

(ii) all residues $\left(x^{*}, x\right)_{\sigma}$ have a bound of the form $K|x|\left|x^{*}\right|$.

\section{REFERENCES}

1. N. Dunford, Spectral Theory I, Convergence to projections, Trans. Amer. Math. Soc. 54 (1943), $185-217$.

2. W. Orlicz, Beiträge zur Theorie der Orthogonalentwicklungen II, Studia Math., 1 (1929), 241 - 255; in particular, pp. $244-247$.

3. A. E. Taylor, The resolvent of a closed transformation, Bull. Amer. Math. Soc. 44 (1938), 70 - 74.

YALE UNIVERSITY 


\section{PACIFIC JOURNAL OF MATHEMATICS}

\section{EDITORS}

\section{R. M. RoBINSON \\ University of California Berkeley 4, California}

\author{
*R. P. Dilmorth \\ Califomia Institute of Technology \\ Pasadena 4, California
}

E. F. BeckenbaCh, Managing Editor

University of California

Los Angeles 24, California

${ }^{*}$ During the absence of Herbert Busemann in 1952.

\section{ASSOCIATE EDITORS}

$\begin{array}{llll}\text { R. P. DILWORTH } & \text { P. R. HALMOS } & \text { BØRGE JESSEN } & \text { J. J. STOKER } \\ \text { HERBERT FEDERER } & \text { HEINZ HOPF } & \text { PAUL LÉVY } & \text { E. G. STRAUS } \\ \text { MARSHALL HALL } & \text { R. D. JAMES } & \text { GEORGE PÓLYA } & \text { KÖSAKU YOSIDA }\end{array}$

\section{SPONSORS}

UNIVERSITY OF BRITISH COLUMBIA

CALIFORNIA INSTITUTE OF TECHNOLOGY

UNIVERSITY OF CALIFORNIA, BERKELEY

UNIVERSITY OF CALIFORNIA, DAVIS

UNIVERSITY OF CALIFORNIA, LOS ANGELES

UNIVERSITY OF CALIFORNIA, SANTA BARBARA

OREGON STATE COLLEGE

UNIVERSITY OF OREGON

\author{
UNIVERSITY OF SOUTHERN CALIFORNIA \\ STANFORD UNIVERSITY \\ WASHINGTON STATE COLLEGE \\ UNIVERSITY OF WASHINGTON \\ AMERICAN MATHEMATICAL SOCIETY \\ NATIONAL BUREAU OF STANDARDS, \\ INSTITUTE FOR NUMERICAL ANALYSIS
}

Mathematical papers intended for publication in the Pacific Journal of Miathematics should be typewritten (double spaced), and the author should keep a complete copy. Manuscripts may be sent to any of the editors. All other communications to the editors should be addressed to the managing editor, E. F. Beckenbach, at the address given above.

Authors are entitled to receive 100 free reprints of their published papers and may obtain additional copies at cost.

The Pacific Journal of Mathematics is published quarterly, in March, June, September, and December, by the University of California, Berkeley 4, California. The price per volume (4 numbers) is \$8.00; single issues, $\$ 2.50$. Special price to individual faculty members of supporting institutions and to individual members of the American Mathematical Society: $\$ 4.00$ per volume; single issues, $\$ 1.25$.

Subscriptions, orders for back numbers, and changes of address should be sent to the publishers, University of California Press, Berkeley 4, California.

Printed at Ann Arbor, Michigan. Entered as second class matter at the Post Office, Berkeley, California.

\section{UNIVERSITY OF CALIFORNIA PRESS • BERKELEY AND LOS ANGELES}




\section{Pacific Journal of Mathematics}

\section{Vol. 2, No. $4 \quad$ April, 1952}

Shmuel Agmon, On the singularities of Taylor series with reciprocal coefficients .................................... 431

Richard Arens, A generalization of normed rings ............... 455

Iacopo Barsotti, Intersection theory for cycles of an algebraic variety . . . . 473

Leonard M. Blumenthal, Two existence theorems for systems of linear inequalities ..................................... 523

Frank Herbert Brownell, III, Translation invariant measure over separable Hilbert space and other translation spaces................. 531

J. W. S. Cassels, On a paper of Niven and Zuckerman ............... 555

Nelson Dunford, Spectral theory. II. Resolutions of the identity .......... 559

Eugene Lukacs and Otto Szász, On analytic characteristic functions ...... 615

W. A. Mersman, Evaluation of an integral occurring in servomechanism theory.......................................... 627

Lawrence Edward Payne and Alexander Weinstein, Capacity, virtual mass, and generalized symmetrization......................... 633

Choy-Tak Taam, The boundedness of the solutions of a differential equation in the complex domain ...................................... 643 